\title{
Aurore: A platform for ultrafast sciences
}

\author{
N. Fedorov, ${ }^{1}$ S. Beaulieu, ${ }^{1, a)}$ A. Belsky, ${ }^{1}$ V. Blanchet, ${ }^{1, b)}$ R. Bouillaud, ${ }^{1}$ M. De Anda Villa, ${ }^{2}$ A. Filippov, ${ }^{1}$ C. \\ Fourment, ${ }^{1}$ J. Gaudin, ${ }^{1, c)}$ R.E. Grisenti, ${ }^{3}$ E. Lamour, ${ }^{2}$ A. Lévy, ${ }^{2}$ S. Macé, ${ }^{2}$ Y. Mairesse, ${ }^{1, d)}$ P. Martin, ${ }^{1}$ P. \\ Martinez, ${ }^{1}$ P. Noé, ${ }^{4}$ I. Papagiannouli, $\left.{ }^{1},{ }^{e}\right)$ M. Patanen, ${ }^{5}$ S. Petit, ${ }^{1}$ D. Vernhet, ${ }^{2}$ K. Veyrinas, ${ }^{1}$ and D. Descamps ${ }^{1, f)}$ \\ 1) Université de Bordeaux - CNRS - CEA, CELIA, UMR5107, F-33405 Talence, \\ France \\ ${ }^{2)}$ Sorbonne Université, CNRS, UMR 7588, Institut des Nanosciences de Paris, INSP, Campus Pierre et Marie Curie, \\ F-75252 Paris Cedex 05, France \\ ${ }^{3)}$ Institut für Kernphysik, J. W. Goethe Universität, Max von Laue str. 1, 60438 Frankfurt am Main, \\ Germany \\ 4) Université Grenoble Alpes, CEA-LETI, 17 rue des Martyrs, F-38054 Grenoble Cedex 9, \\ France \\ 5) Nano and Molecular Systems Research Unit, Faculty of Science, University of Oulu, P.O. Box 3000, FI-90014 Oulu, \\ Finland
}

(Dated: 11 August 2020)

We present the Aurore platform for ultrafast sciences. This platform is based on a unique 20 $\mathrm{W}, 1 \mathrm{kHz}, 26$ fs Ti:Sapphire laser system designed for reliable operation and high intensity temporal contrast. The specific design ensures a high stability in terms of pulse duration, energy and beam pointing necessary for extended experimental campaigns. The laser supplies 5 different beamlines, all dedicated to a specific field: attosecond science (Aurore 1), ultrafast phase transitions in solids (Aurore 2 and 3), ultrafast luminescence in solids (Aurore 4), femtochemistry (Aurore 5). The technical specifications of these five beamlines are described in details and examples of recent results are given.

PACS numbers: Valid PACS appear here

Keywords: Suggested keywords

\section{INTRODUCTION}

Since the first demonstrations of laser with titaniumsapphire (Ti:Sapphire, $\mathrm{Ti}^{3+}: \mathrm{Al}_{2} \mathrm{O}_{3}$ ) in the early eighties $^{1-3}$, this material has been one of the major building brick of the ultrafast science. Taking advantage of the chirped pulse amplification technique, ${ }^{4}$ Ti:Sapphire lasers nowadays span pulses duration vs energy space ranging from few femtosecond ${ }^{5}$ with carrier envelop phase (CEP) stabilization and $\mathrm{mJ}$ up to $\mathrm{kJ}$ pulse reaching several PW peak intensities. This versatility makes Ti:Sapphire lasers particularly suitable for secondary sources ranging from $\mathrm{THz}$ to $\mathrm{x}$-ray wavelengths, extending the field of science that can be explored and notably to the attosecond timescale thanks to high-harmonics generation. In a nutshell: Ti:Sapphire lasers are the swiss knives of the ultrafast science community.

As a result, femtosecond lasers enable the investigation of ultrafast dynamics in matter, and have led to important

\footnotetext{
a) Now at: Fritz Haber Institute of the Max Planck Society, Department of Physical Chemistry, 14195 Berlin, Germany

b) Electronic mail: Author to whom correspondence should be addressed: valerie.blanchet@u-bordeaux.fr

c) Electronic mail: Author to whom correspondence should be addressed: jerome.gaudin@u-bordeaux.fr

d) Electronic mail: Author to whom correspondence should be addressed: yann.mairesse@u-bordeaux.fr

e) Now at: Laboratoire de Physique des Gaz et des Plasmas, Université Paris-Saclay, CNRS, UMR8578, F-91405 Orsay Cedex, France f)Electronic mail: Author to whom correspondence should be addressed: dominique.descamps@u-bordeaux.fr
}

breakthroughs in the past decades. The broad range of applications leads to a variety of requirements, depending on the nature of the target (solid, liquid, gaseous), the excitation and/or the probe wavelengths (from the midIR to the extreme ultraviolet), and the timescale of the dynamics (picoseconds, femtoseconds, attoseconds). The Aurore platform at the CEntre Lasers Intenses et Applications (CELIA) was designed to fulfill all these requirements used in numerous experiments ranging from solid state physics $^{6}$, warm-dense matter ${ }^{7}$, femtochemistry ${ }^{8}$ and attosecond science ${ }^{9}$ as will be shown later on. Its core is a $20 \mathrm{~W}$ Ti:Sapphire laser delivering $26 \mathrm{fs}$ pulses at $1 \mathrm{kHz}$ that can supply up to three experiments in parallel. Other similar platforms driven by a $\mathrm{kHz}$ and multi-m J class femtosecond laser exist in the world, for instance, the Artemis facility at the Central Laser Facility (UK), the Attolab $\mathrm{kHz}$ laser of the Lund Laser Center (Sweden), the ATTOLab platform of Paris-Saclay university (France), the Allegra ELI beamlines (Cezch Republic) Only Allegra laser facility is based on optical parametric amplification (OPA) while the three others laser facilities use the Ti:Sapphire technology. To portray a picture of what is currently available with this kind of laser facilities, we describe in Table 1 the characteristics of the primary and secondary sources as well as the end-station equipment for the four platforms mentioned above.

Five beamlines are devoted to different applications, using various frequency conversion schemes and optical or photoelectrons / photoions diagnostics. In this paper, we present the Aurore Platform dedicated to ultrafast dynamics of matter. The first part of the manuscript describes the Aurore laser system. The following parts focus on each beamline, discussing their peculiarities, each illustrated by one recent application. 
TABLE I. Compilation of $\mathrm{kHz} / \mathrm{mJ}$ laser class facilities, i.e. open to external users. Data are extracted from web sites and references. List of used acronyms: OPA: optical parametric amplifier, ARPES: angle-resolved photoelectron spectroscopy, HHG: high-harmonic generation, VMI: velocity map imaging, TOF: time of flight, PES: photolectron spectroscopy, TR-SR-PES: time and spin-resolved photoelectron spectroscopy, CEP: carrier envelope phase, CDI: coherent diffractive imaging, TR-SE: timeresolved spectroscopic ellipsometry, TR: time-resolved, XRD: x-ray diffraction, XAS: x-ray absorption spectroscopy, XES: x-ray emission spectroscopy

\begin{tabular}{|c|c|c|c|}
\hline Facility & Laser parameters & Secondary light sources & beamlines and/or end-stations \\
\hline $\begin{array}{c}\text { Artemis } \\
\text { Central Laser Facility }\end{array}$ & $\begin{array}{c}30 \mathrm{fs}-2 \times 8 \mathrm{~mJ} \\
780 \mathrm{~nm}-1 \mathrm{kHz}\end{array}$ & $\begin{array}{c}\text { OPA }(235 \mathrm{~nm} \text { to } 15 \mu \mathrm{m}) \\
\text { fs post compressed } 780 \mathrm{~nm} \\
\text { fHG }(10-100 \mathrm{eV})\end{array}$ & $\begin{array}{c}\text { Condensed matter: ARPES + TR-SR-PES } \\
\text { Gas Phase: VMI/TOF } \\
\text { XUV Imaging } \\
\text { liquid microjet }\end{array}$ \\
\hline $\begin{array}{c}\text { Attolab kHz laser } \\
\text { Lund Laser Centre }\end{array}$ & $\begin{array}{c}20 \mathrm{fs}-5 \mathrm{~mJ} \\
800 \mathrm{~nm}-1 \mathrm{kHz} \\
\text { CEP stable }\end{array}$ & $\begin{array}{c}\text { OPA }(1100-1800 \mathrm{~nm}) \\
\text { HHG }(17-150 \mathrm{eV})\end{array}$ & Gas Phase: TOF, VMI \\
\hline $\begin{array}{c}\text { ATTOLab } \\
\text { Laboratoire Interactions, } \\
\text { Dynamique et Lasers }\end{array}$ & $\begin{array}{c}800 \mathrm{~nm}-15 \mathrm{~mJ} \\
\text { CEP stable }\end{array}$ & $\begin{array}{c}\text { OPA }(1200-2000 \mathrm{~nm}) \\
\text { HHG }(15-100 \mathrm{eV})\end{array}$ & Gas Phase: TOF, VMI \\
\hline $\begin{array}{c}\text { Laser 1 Allegra } \\
\text { ELI beamlines }\end{array}$ & $\begin{array}{c}15 \mathrm{fs}-20 \mathrm{~mJ} \\
835 \mathrm{~nm}-1 \mathrm{kHz}\end{array}$ & $\begin{array}{c}\text { plasma X-ray }(1-77 \mathrm{keV}) \\
\text { HHG }(10-120 \mathrm{eV})\end{array}$ & $\begin{array}{c}\text { AMO VMI/TOF and CDI } \\
\text { Solid state: NIR to NUV TR-SE } \\
\text { TR-XAS/XES, TR-XRD } \\
\text { Raman, optical absorption spectroscopy }\end{array}$ \\
\hline
\end{tabular}

\section{THE AURORE LASER FACILITY}

The Aurore laser is based on chirped pulse amplification (CPA) scheme in Ti:Sapphire amplifiers (cf. Fig. 1). The first brick of the laser is a Kerr-lens modelocked Ti:Sapphire oscillator (Femtolasers, Fusion Pro) which delivers $420 \mathrm{~mW}, 12 \mathrm{fs}$ pulses at $75 \mathrm{MHz}$. To counteract spectral gain-narrowing in Ti:Sapphire amplifiers without reducing the available seed energy, intracavity spectral shaping is achieved in the oscillator resulting in a M-shaped output spectrum with $30 \%$ amplitude modulation and a bandwidth of $84 \mathrm{~nm}$ (FWHM). The oscillator pulses are then stretched to 510 ps by an all-reflective stretcher design based on an aberration free Öffner triplet ${ }^{10}$ with 1200 grooves/mm grating. As the amplified bandwidth gets broader, significant spectral phase distortions are present (group velocity dispersion of mirrors, polarizers, Pockels cells and Ti:Sapphire amplifiers) and need to be corrected to provide clean recompressed pulses. To do so, an acousto-optic programmable dispersive filter (Dazzler, FASTLITE) is inserted at the stretcher output and coupled with a temporal pulse characterization (Self-Referenced Spectral Interferometry, Wizzler, FASTLITE) performed at the Aurore compressor output with microjoule pulse energy. In addition, the Dazzler device enables fine spectral shaping of the seed pulse to provide day after day identical characteristics of the amplified pulse spectrum.

At the Dazzler output, a potassium dihydrogen phosphate (KDP) Pockels cell (PC piker) is used to reduce the repetition rate down to $1 \mathrm{kHz}$ with an extinction ratio higher than $10^{4}$. The chirped pulses are first amplified in a regenerative Ti:Sapphire amplifier pumped by a Q-switched frequency-doubled neodymium-doped yttrium lithium fluoride (Nd:YLF) laser $(1 \mathrm{kHz}, 18 \mathrm{~W}$ at $527 \mathrm{~nm}$, Jade2 - Thales) up to $1.8 \mathrm{~mJ} /$ pulse. To remove parasitic reflections from the intracavity broadband polarizer of the regenerative amplifier and then improve pulse intensity contrast in nanosecond time scale, a sec- ond KDP Pockels cell (PC cleaner) is used before further amplification. The second and last Ti:sapphire amplifier is cooled by a high power pulse tube cryorefrigerator (PT90, Cryomech) to reduce the thermal lensing ${ }^{11}$ induced by the heat load of the pump power provided by 4 Q-switched frequency-doubled Nd:YLF lasers $(1 \mathrm{kHz}$, $28 \mathrm{~W}$ at $527 \mathrm{~nm}$, Jade2 - Thales). By keeping the temperature as low as $-170^{\circ} \mathrm{C}$, the thermal focal lens in the Ti:Sapphire crystal reaches a dozen meters, enabling a multi-pass amplifier architecture without using focusing optics, i.e. with free beam propagation. At each pass in the Ti:Sapphire crystal, the spatial overlap between pump and signal beams is ensured by the combination of the natural beam divergence of the IR (increase beam size with length of the amplifier arms) and the radial optical gain of the Ti:Sapphire amplifier (Gaussian distribution) reshaping the beam waist size. After 4 passes in the cryo-cooled Ti:sapphire crystal, the pulse energy is amplified up to $22 \mathrm{~mJ} /$ pulse with a spectral bandwidth of $54 \mathrm{~nm}$ (FWHM) and a central wavelength of $800 \mathrm{~nm}$ (Fig.2 (c)). At the output of this last amplifier, the beam pointing is stabilized (to mainly compensate slow drifts ) by an active device (Aligna, Messtechnik).

Finally, the laser beam diameter is expanded to 25 $\mathrm{mm}\left(\right.$ at $\mathrm{I}_{\max } / \mathrm{e}^{2}$ ) and distributed among five beamlines with up to 3 simultaneously, each of these dedicated to a specific field of research. The main characteristics of these beamlines are summarized in Table 1. As a beamline can be up to $15 \mathrm{~m}$ long, the pulses propagate stretched and are recompressed close to each experimental set-ups by a double-pass Treacy compressor ${ }^{12}$ using 1480 grooves/mm gratings and ensuring a total transmission of $70 \%$. The use of mismatched grating strecher/compressor (1200 grooves/mm versus 1480 grooves $/ \mathrm{mm}$ ) results in a better compensation of the material dispersion of amplifier optics ${ }^{13}$. The temporal pulse profile at the compressor output is characterized by a Second-Harmonic Frequency-Resolved Optical Gating (SHG-FROG) device. Figure 2 shows the FROG 


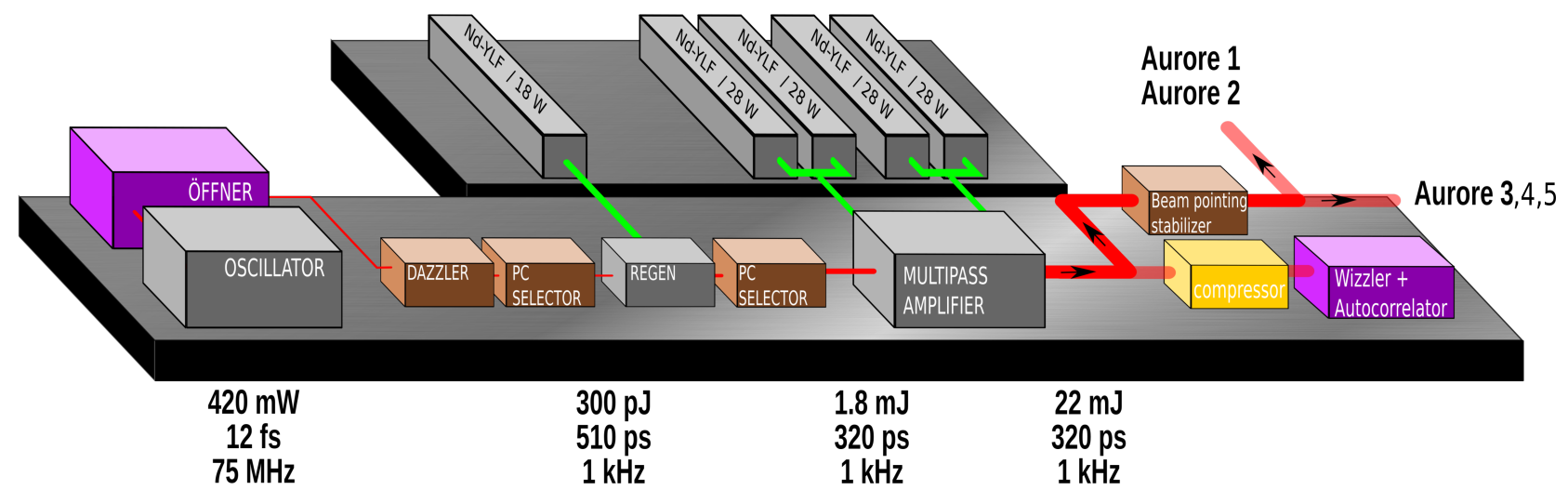

FIG. 1. Schematic view of the Aurore laser system with its main constituting blocks
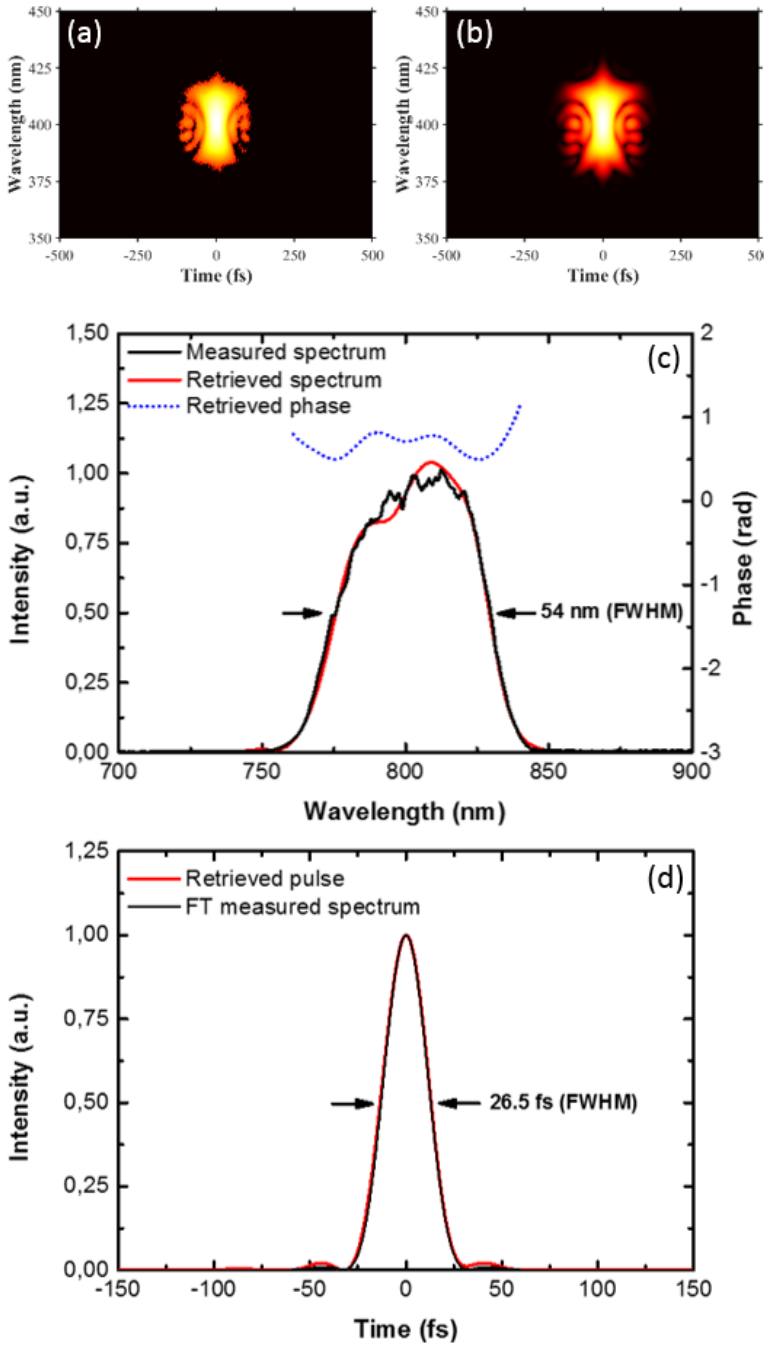

FIG. 2. Retrieved intensity profile of the output pulses from SHG-FROG device. (a) Measured SHG-FROG trace. (b) Retrieved SHG-FROG trace. (c) Retrieved SHG-FROG spectrum (black curve) and phase (dashed blue curve) superimposed with the experimental output pulse spectrum (red curve). (d) Retrieved intensity profile (red curve) superimposed with the Fourier-transform of the experimental spectrum (black curve).

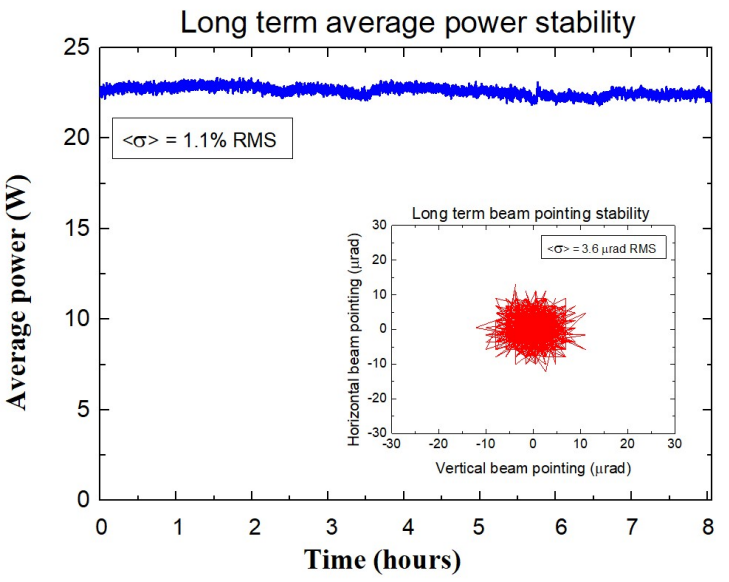

FIG. 3. Long-term stability of the Aurore laser. Measurements of the average power and the beam pointing (inserted graph) of the laser during 8 hours.

traces and the retrieved spectrum and intensity profile at the compressor output of the Aurore beamline 3 . A pulse duration of 26.5 fs (FWHM) is achieved very close to the Fourier-transform limitation of the output pulse spectrum (Fig.2 (d)). As many experiments use secondary sources and require acquisition time of up to several hours, the long-term stability of the Aurore laser characteristics is prime of importance. Particular care has therefore been taken to i) reduce the mechanical vibration level on or near the optical tables ii) choose the most stable optical mounts with a laser beam height not exceeding $50 \mathrm{~mm}$ iii) stabilize the ambient temperature of the laser room at $\pm 0.5^{\circ} \mathrm{C}$ iv) avoid or water-cool heat sources on the optical tables. Thus, after one hour of warm-up, the long term stability (measurements over 8 hours) of the Aurore laser facility is typically of $1.1 \%$ (RMS) in average power and $3.6 \mu \mathrm{rad}(\mathrm{RMS})$ in beam pointing (Fig. 3).

A very important parameter while performing condensed matter experiments with ultra-short lasers is the temporal pulse contrast. Indeed a low contrast results in a pre-heating effect of the sample which can strongly disturb the experiment, especially for material 


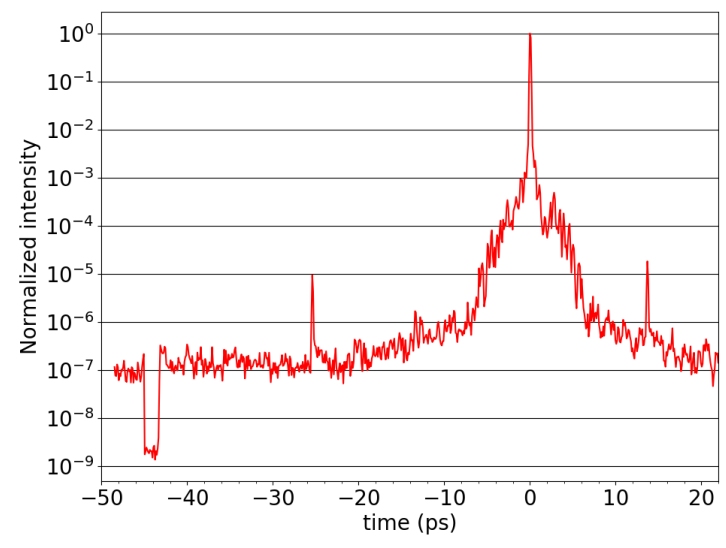

FIG. 4. High dynamic range temporal contrast measurement of the compressed pulses carried out with a third-order autocorrelator.

with very low temperature of fusion. In Fig. 4 we show a typical third-order autocorrelator measurement (SEQUOIA system, Amplitude Technologies). The two visible peaks at $-24 \mathrm{ps}$ and 12 ps are replicas from the third-order autocorrelator apparatus, while the feature at -44 ps obtained without the beam shows the background level of the measurement. The pedestal of the compressed pulse in the $10 \mathrm{ps}$ time window exists in all CPA laser systems using large pulse stretching factor (grating-based stretcher) and results from the scattering light induced by the grating of the stretcher ${ }^{14,15}$. The signal at $10^{-7}$ is the amplified spontaneous emission (ASE) level which lasts around $3 \mathrm{~ns}$ and corresponds to nearly $1.5 \%$ of the fs pulse in energy. The high contrast of Aurore makes this source highly suitable to avoid pre-heating in condensed phase.

\section{BEAMLINE 1: ATTOSCIENCE}

One of the greatest advantages of the Aurore 1 beamline is versatility. Indeed, the different building blocks available in the experimental hall (XUV beamline and spectrometers, Velocity Map Imaging spectrometer, fewcycle pulse generation module, various molecular beam sources) can be reconfigured on demand for different applications.

\section{A. High-order harmonic generation in gas phase}

The Aurore 1 beamline is mostly dedicated to attosecond science and molecular spectroscopy using high-order harmonic generation (HHG). The HHG process occurs when laser pulses are focused at intensities around $10^{14}$ $\mathrm{W} \cdot \mathrm{cm}^{-2}$ in an atomic or molecular gas. The strong laser field lowers the Coulomb potential of the target, enabling electrons to tunnel out in the continuum. The freed electron wavepacket is then accelerated by the laser field, gaining a high amount of kinetic energy. When the laser field reverses, the electrons can be driven back to their parent ion and radiatively recombine, emitting an attosecond burst of extreme ultraviolet light. This process repeats every half laser period, creating a spectrum made of odd harmonics of the fundamental laser frequency.

Since its discovery in $1987^{16,17}$, HHG has been used in many experiments as a source of coherent femtosecond and attosecond light pulses in the extreme ultraviolet. Indeed, HHG sources possess a number of properties that make them ideal for many applications: excellent spatial coherence ${ }^{18}$, high brightness (above $10^{10}$ photons per pulse using energetic Ti:Sapphire laser pulses ${ }^{19,20}$, above $10^{13}$ photons per second using high-repetition rate Ytterbium fiber lasers ${ }^{21,22}$ ), and ultrashort duration (femtosecond ${ }^{23,24}$ and attosecond ${ }^{25-27}$ pulses). These properties make HHG sources appealing for timeresolved measurements in atomic, molecular, and solidstate physics, but also as a simple alternative Vacuum UltraViolet source of irradiation to investigate laboratory astrochemistry, for instance ${ }^{28}$.

In parallel to these XUV source developments, a new route for applications of HHG emerged in the early 2000 's, once it was realized that the high-harmonic emission encodes structural and dynamical information on the generating medium ${ }^{29,30}$. This resulted in a new class of measurements, referred to as high-harmonic spectroscopy or in-situ measurements, in which strong-field driven attosecond electron wavepackets are used to probe the system under investigation. The structural and dynamical informations are encoded in the spectrum, spectral phase, and polarization state of the emitted XUV radiation.

\section{B. General features of the experimental set-up}

The Aurore 1 beamline is a versatile set-up, which can be reconfigured on demand for different applications. The main ingredients are the following:

Postcompression: The 26 fs pulse duration of the Aurore laser is shortened down to $7 \mathrm{fs}$ (FWHM) in a postcompression module based on the self-phase modulation in a noble gas-filled hollow core fiber. To reach millijoule ouput energy level, a stretched fiber with a $500 \mu \mathrm{m}$ inner diameter and $1.5 \mathrm{~m}$ length is used and filled with a pressure gradient of neon ${ }^{31,32}$. At the fiber output, the beam is collimated in vacuum chamber to a diameter of $6 \mathrm{~mm}\left(I_{\max } / e^{2}\right)$ and sent to six pairs of chirped mirrors (-50 $\mathrm{fs}^{2}$ per bounce) and silica plates (thickness $\sim 5 \mathrm{~mm}$ ) to compensate the group delay dispersion. In this configuration, we are able to daily produce pulses of $7 \mathrm{fs}$ $1.2 \mathrm{~mJ}$ when 1.1 bar of neon pressure is used.

Optical frequency conversion: The spectral tunability of the laser pulses is rather limited in Aurore 1, with basic nonlinear frequency conversion set-ups available to produce second $(400 \mathrm{~nm})$ and third $(267 \mathrm{~nm})$ harmonic radiation.

High-order harmonic generation: Different HHG schemes can be implemented. For applications requiring high XUV flux, the laser or its second harmonic is loosely focused in a gas cell filled with krypton, acetylene, argon 
TABLE II. Summary of the different Aurore beamlines.

List of used acronyms: NOPA: non-colinear optical parametric amplifier, UPS: ultraviolet photoelectron spectroscopy, XPS: x-ray photoelectron spectroscopy

\begin{tabular}{|c|c|c|c|c|}
\hline Beamline & Topics & $\begin{array}{c}\text { Number of } \\
\text { compressors }\end{array}$ & Secondary light sources & Experimental end-stations \\
\hline BL 1 & Attoscience & 1 & $\begin{array}{c}\text { HHG }(20-100 \mathrm{eV}) \\
7 \mathrm{fs} 1.2 \mathrm{~mJ} \text { post-compressed pulses }\end{array}$ & $\begin{array}{c}\text { XUV spectroscopy and polarimetry } \\
\text { Velocity map imaging }\end{array}$ \\
\hline BL 2 & Solid state & 2 & NOPA $380-680 \mathrm{~nm} 80 \mathrm{fs}-3-4 \mu \mathrm{J}$ pulse & $\begin{array}{c}\text { Frequency domain interferometry } \\
\text { on bulk/thin film samples up to } 9 \text { ps }\end{array}$ \\
\hline BL 3 & Solid state & 2 & $\begin{array}{c}\text { HHG monochromatized }(75-95 \mathrm{eV}) \\
\text { multi-layer based monochromator }\end{array}$ & Hemisperical analyzer UPS and XPS \\
\hline BL 4 & Solid state & 2 & $\begin{array}{c}\text { HHG monochromatized }(20-50 \mathrm{eV}) \\
\text { grating based monochromator }\end{array}$ & Photo-luminescence \\
\hline BL 5 & $\begin{array}{c}\text { Small } \\
\text { quantum } \\
\text { systems }\end{array}$ & 1 & $\begin{array}{c}\text { UV NOPA, tunable from } \\
750 \text { nm to } 200 \mathrm{~nm} \text {, at few } \mu \mathrm{J} / p, \\
\text { with duration varying from } 50 \text { to } 130 \mathrm{fs}\end{array}$ & $\begin{array}{c}\text { Velocity map imaging of charged particles } \\
8 \% \text { energy resolution up to } 17 \mathrm{eV} \\
\text { ion mass spectroscopy with } \Delta m / m=0.7 \%\end{array}$ \\
\hline
\end{tabular}

or neon depending on the target spectral range (from 15 $\mathrm{eV}$ to $100 \mathrm{eV}$ ). The typical photon flux produced in the $15-30 \mathrm{eV}$ range is $10^{11}$ photons/s per harmonic. Quasicircularly polarized XUV radiation can be produced in specific spectral ranges, by using resonant high-order harmonic generation in $\mathrm{SF}_{6}$ or argon ${ }^{33,34}$ gas jet.

For high-harmonic spectroscopy, a $100 \mathrm{~Hz}$ pulsed General Valve or a $1 \mathrm{kHz}$ pulsed Even-Lavie valve can be used to produce harmonics in cold molecular beams ${ }^{35}$ or clusters $^{36}$. Liquid or solid samples can be introduced inside the Even-Lavie valve, heated, and pushed by a backing pressure of rare gas to produce a seeded gas jet. Alternatively, they can be placed in an oven connected to a static cell under vacuum. This scheme was, for instance, used to investigate high-harmonic emission from chiral fenchone molecules ${ }^{37}$. Sophisticated pump-probe geometries can be implemented with any relative polarizations between the laser beams. Transient grating spectroscopy uses two non-colinear pumps and one probe beam, enabling high-contrast measurements. We used this scheme to follow the photodissociation of $\mathrm{NO}_{2}$ molecules ${ }^{38}$. Inserting a phase mask in the generating laser beams produces two spatially separated harmonic sources, which interfere in the far field, enabling phase-resolved measurements of the XUV emission ${ }^{35,39}$. It is even possible to combine transient gratings and two-sources interferometry to obtain information on the vectorial properties of the high-harmonic emission, e.g. when generating from aligned molecules ${ }^{35}$.

XUV optics: High-order harmonic generation in gas phase produces broad spectra constituted of odd harmonics of the fundamental frequency (separated by 3.1 $\mathrm{eV}$ when using a $800 \mathrm{~nm}$ pulse). The spectral splitting between two consecutive harmonics can be broadened by decreasing the driving wavelength, to reach $6.2 \mathrm{eV}$ when using a $400 \mathrm{~nm}$ driver. Many applications require a spectral selection. In Aurore 1, this is achieved by using metallic foils, which play the role of bandpass filters $(\mathrm{Zr}$ around $26 \mathrm{eV}, \mathrm{Al}$ : 20-70 eV, In around $15 \mathrm{eV}$ and $\mathrm{Sn}$ around $22 \mathrm{eV}$, Luxel filters). The XUV radiation can be refocused by gold-coated toroidal mirror with $500 \mathrm{~mm}$ focal distance under $85^{\circ}$ grazing incidence (Winlight) to focus this HHG emission into a velocity map imaging (VMI) for instance. The alignment of this toroidal mir- ror is motorized to properly minimize the astigmatism at the focus, while maintaining a spatial overlap with a molecular beam and a second laser pulse for instance ${ }^{40}$. Detectors:

The XUV radiation is analyzed by a flat-field XUV spectrometer, consisting of a $1200 \mathrm{~mm}^{-1}$ variable groove spacing spherical grating used under grazing incidence and a set of dual microchannel plates with a rapid phosphor screen (P46, decay time of $99 \%$ within $\sim 1 \mu \mathrm{s}$ ) which allows to measure single-shot HHG spectra at 1 $\mathrm{kHz}$. A 12-bit cooled CCD camera is used to image the phosphor screen and to record the spatially resolved harmonic spectra, enabling for instance to resolve subtle effects such as spectro-spatial rings in the HHG process ${ }^{41}$. The XUV polarization state can be partially analyzed by a rotating polarizer consisting of bare gold mirrors, which provides the polarization direction and an upper bound of the ellipticity.

Moreover, photoionization experiments can be carried out in a VMI Spectrometer, consisting of a gas jet produced by a continuous jet or $1 \mathrm{kHz}$ pulsed Even Lavie valve followed by a skimmer, a set of high-voltage electrodes which image the projection of the 3D photoelectron or photoion angular distribution onto a set of dual microchannel plates associated with a P43 phosphor screen and 12-bit SCMOS camera. This set-up was recently used to investigate photoelectron circular dichroism from chiral molecules ${ }^{42}$ and to track attosecond delays in chiral photoionization ${ }^{43}$.

\section{Single-shot CEP-tagging using the Attosecond lighthouse}

One of the great challenges in attosecond science is to be able to gate the HHG emission in order to produce an Isolated Attosecond Pulse (IAP) out of an Attosecond Pulse Train (APT), which naturally emerges from HHG driven by multicycle laser pulse. Different gating schemes have been developed in the past few years to temporally confine the high-harmonic emission to a single optical half-cycle, resulting in the production of an IAP-ionization gating ${ }^{44}$, amplitude gating ${ }^{45}$, polarization gating ${ }^{46}$ and double optical gating ${ }^{47}$. In 2012, 


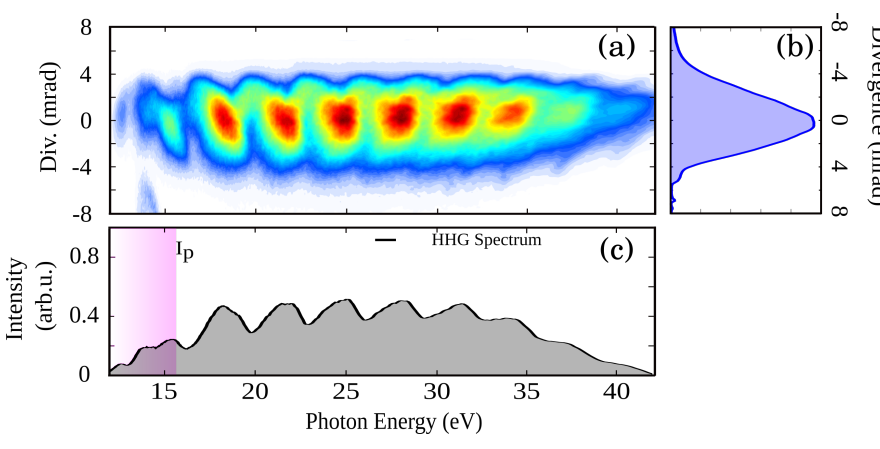

FIG. 5. Attosecond lighthouse technique using CEP-unlocked laser. (a) Spatially-resolved harmonic spectrum driven by a spatially chirped laser pulse of 7 fs. (b) Spectrally integrated harmonics spatial profile. (c) Spatially integrated harmonics spectrum.

Vincenti and Quéré ${ }^{48}$ proposed a new idea based on the use of space-time couplings in ultrashort light beams: the attosecond lighthouse.

The attosecond lighthouse effect results of a controlled space-time coupling on the wavefront of the fundamental beam, such that consecutive attosecond bursts from the train are emitted in different directions ${ }^{48}$. By inserting a pair of well-misaligned wedges in a collimated beam, an angular dispersion is introduced in the fundamental beam, which gives rise to a spatial chirp at the focus: the blue and red edges of the laser spectrum are focused into different places, e.g. vertically shifted. This spatial chirp induces an ultrafast wavefront rotation, meaning that the wavefronts for different consecutive laser half-cycles are not parallel. Because XUV bursts are emitted perpendicular to the laser wavefront, consecutive attosecond bursts propagate in different directions. In combination with spatial filtering in the far-field, the attosecond lighthouse effect can thus be used to produce a source of multiple IAP. Recently, Hammond et $a l^{49}$ performed a temporal characterization (streaking measurement) of the three most intense beamlets generated by the attosecond lighthouse, and confirmed that they were isolated attosecond pulses.

In an attosecond lighthouse experiment, the propagation direction of the beamlets depends also on the carrier-envelop phase (CEP) of the laser pulse. Thus, one needs to have a CEP-stabilized laser in order to send the beamlets in the same direction from pulse-to-pulse. On the others hand, in their seminal paper, Vincenti and Quéré formulated the idea of using the single-shot far-field profile of the XUV emitted from the attosecond lighthouse to perform an in situ tagging of the CEP of the laser ${ }^{48}$. Such a tagging releases the constraints on the laser source, enabling CEP-resolved experiments without CEP stabilization.

In order to illustrate the possibility of CEP-resolved experiments without CEP stabilization, we used postcompressed pulses from Aurore down to $7 \mathrm{fs}$ and introduced a pair of wedges in the few-cycle laser beam to produce high-order harmonics in a gas jet of argon.

A spatially-resolved high-order harmonic spectrum integrated over 100 laser pulses is shown in Fig. 5(a). The harmonics exhibit large divergence because they are generated from a vertically spatially chirped beam. This is the signature of the angular spreading of the XUV using the attosecond lighthouse. However, the discrete horizontal fringes which result from the angular separation of the different attosecond beamlets along the vertical axis are not visible in this measurement (see Fig. 5(b)). Indeed, the Aurore laser being not CEP-stabilized, the pulse-to-pulse CEP variation leads to the generation of beamlets emitted in different directions. The random CEP fluctuations of the laser thus completely wash out the discrete spatial fringes which are expected to emerge when driving HHG using the attosecond lighthouse setup.

Despite the unstabilized nature of the CEP, it is still possible to perform CEP-resolved measurement if the detector used is able to acquire single-shot spectra. An example of single-shot high-order harmonics spectra recorded for 9 laser pulses is shown in Fig. 6. They show the emergence of horizontal spatial fringes, which are characteristics of angularly spread attosecond bursts of $7 \mathrm{fs}$ pulse. The phase of these spatial fringes is directly related to the CEP of the driving pulse in the generating medium.

Figure 7 presents the single-shot extraction of the phase of the spatial fringes, using Fourier analysis. One can see that over 100 laser pulses, the CEP fluctuations seem to be pretty random. This tagging of the relative in situ CEP in a pulse-to-pulse manner, allows to a posteriori sort the different spectra according to the phase of their spatial fringes $(\Phi)$ and to sum them together to increase the signal-to-noise ratio. In the following, we will sum all the spectra with $-300 \mathrm{mrad}<\Phi<300 \mathrm{mrad}$ (green area in Fig. 7).

The spatially-resolved HHG spectrum of argon generated from the attosecond lighthouse, after CEP sorting, is presented in Fig. 8(a). As explained previously, this spatially-resolved spectrum provides a spatio-temporal mapping of the XUV emission, since the spatial position of the signal on the detector encodes its emission time, with sub-laser-cycle resolution. Well separated horizontal fringes are visible. Since we know that each of these fringes is separated by $1.3 \mathrm{fs}$ (half-cycle of the 800 $\mathrm{nm}$ driving wavelength), they can be used to calibrate the spatio-temporal mapping provided by the attosecond lighthouse effect.

In a recent study, we have demonstrated that the spatio-temporal mapping provided by the attosecond lighthouse enables to investigate near-threshold resonant effects in HHG from Argon ${ }^{50}$. Indeed, using single-shot CEP tagging from a CEP unstable few-cycle laser in the attosecond lighthouse configuration allowed us to demonstrate that HHG coming from the ionization from excited states and recombination to the ground state are delayed by few femtoseconds with respect to non-resonant harmonics. Moreover, we have demonstrated that the absence of spatial fringes on the below threshold free induction decay indicates that this narrowband XUV emission is not confined on the attosecond time-scale. Similarly to the work of Kim et al. ${ }^{51}$, which used the attosecond lighthouse to probe the ionization gating process directly in the time-domain, these measurements extend the scope 

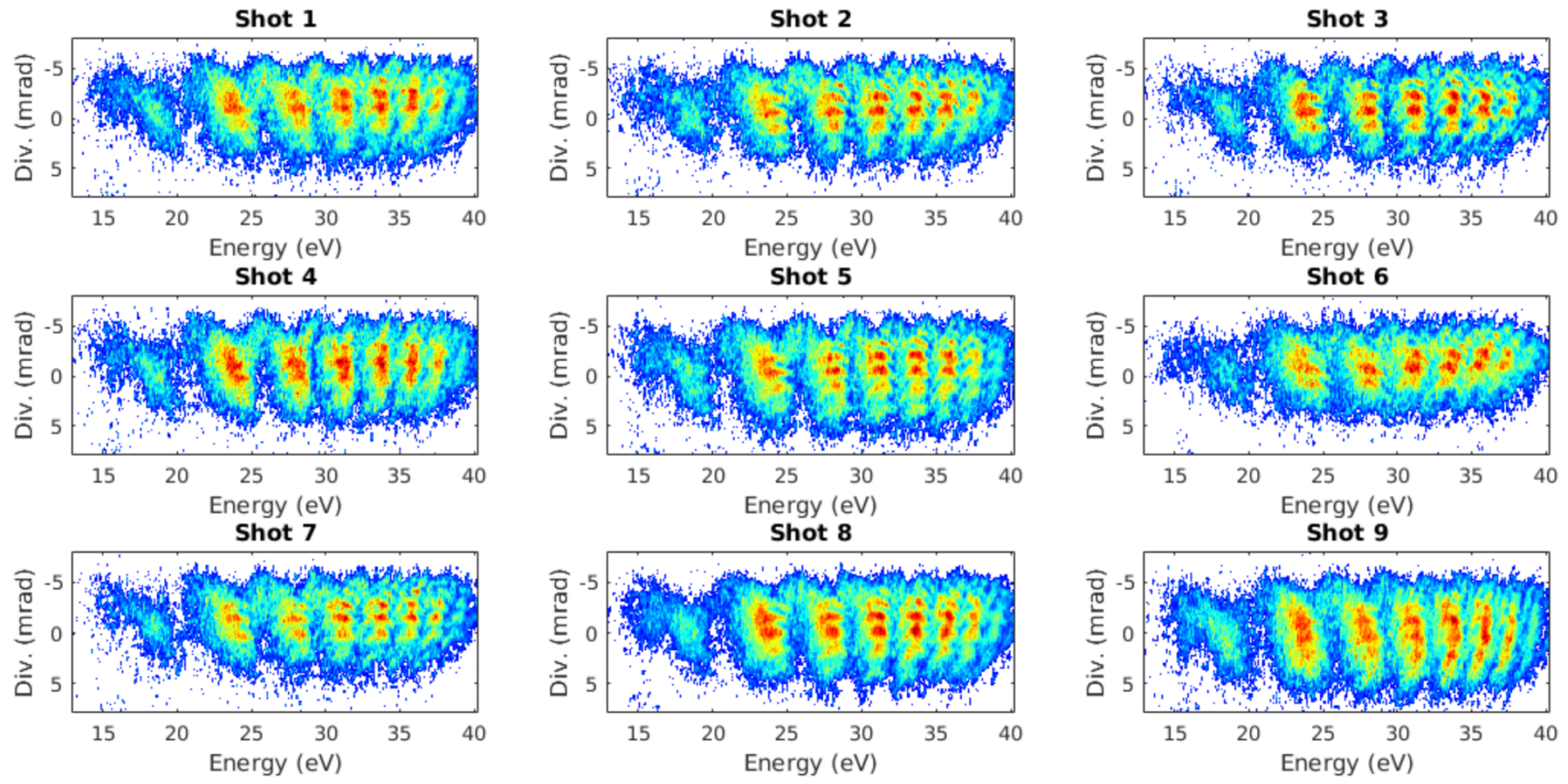

FIG. 6. Nine single-shot measurement of the vertically spatially-resolved high-order harmonics spectrum, when driven by spatially chirped pulses.

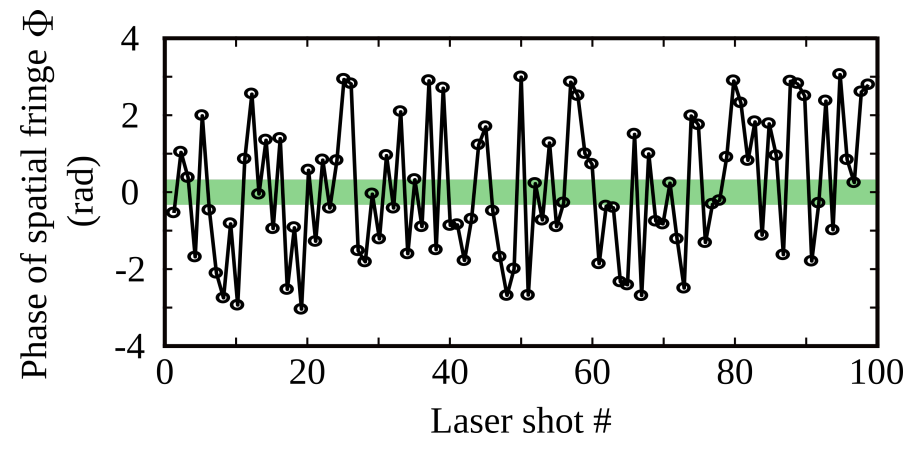

FIG. 7. Phase of the spatial fringes extracted using Fourier analysis, for each laser pulse. The green area represents the window used for CEP sorting the data in order to increase the signal-to-noise ratio as it is in Fig. 8.

of the attosecond lighthouse technique, from a way to generate isolated attosecond pulses to a metrology tool that enables the measurement of ultrafast electronic dynamics during HHG.

\section{BEAMLINE 2: FREQUENCY DOMAIN INTERFEROMETRY FOR PHASE TRANSITION DYNAMICS IN SOLIDS.}

The interaction of sub-picosecond laser pulses with solids leads to the creation of a transient out-ofthermodynamical equilibrium state. In the sub- to few picoseconds following the interaction, the electronic temperature, $T_{e}$, is higher than the atomic one, $T_{i}$. For $T_{e}$ in the order of $1 \mathrm{eV}$, state transition occurs from solid to solid or liquid without reaching the ablation threshold. The fact that $T_{e} \neq T_{i}$ leads to specific phase

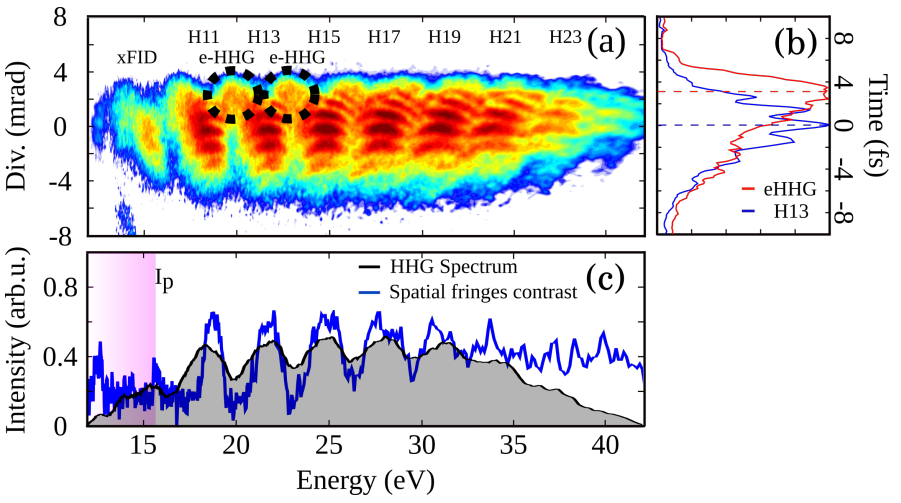

FIG. 8. Time-frequency mapping of HHG using the attosecond lighthouse technique, with a CEP unstable laser, after CEP tagging. (a) Spatially-resolved harmonic spectrum driven by a spatially chirped laser pulse. (b) Spectrally integrated harmonic spatial profile for e-HHG (in red) and for H13 (in blue). The fringe spacing was used to transform the spatial axis into a time axis. (one fringer spacing $=1.3 \mathrm{fs}$ ) (c) Spatially integrated harmonic spectrum (black) and contrast of the spatial fringes (blue). This figure is adapted from ${ }^{50}$.

transition processes often denominated as non-thermal. In covalent materials, non-thermal melting has been predicted and described theoretically while in metallic material, bond hardening process seems at play ${ }^{52}$. The Aurore 2 beamline is dedicated to pump-probe experiments on these phase transitions in solids using the frequency domain interferometry (FDI) technique ${ }^{53}$. The principal advantage of this beamline is the sample holder which allows us to perform experiments at a $\mathrm{kHz}$ repetition rate with an adjustable pump fluence higher than the single-shot laser damage threshold of 
any material.

One of the challenges of this field is to be able to measure microscopic observables (atomic and/or electronic structures) in well characterized macroscopic conditions which require the measurement of $\mathrm{T}_{i}$ and $\mathrm{T}_{e}$. There is no single experimental technique that can provide all these observables at a time. The strength of our set-up is that hydrodynamics of the sample can be retrieved via the $\beta$ parameters, which we will define further on, while the variations of the reflectivity allows us to retrieve the complex dielectric constant $\epsilon$. The hydrodynamics evolution can be related to $\mathrm{T}_{e}$ and $\mathrm{T}_{i}$ using for suitable simulations code ${ }^{54}$, while $\epsilon$ is related to microscopic observable which can be compared to ab-initio simulations performed at the deduced Te and Ti. In this sense we can access, even though indirectly, to the microscopic and macroscopic observables in a single experiment.

\section{A. Two-color, polarization resolved FDI}

Frequency domain interferometry was first demonstrated by Froehly and coworkers ${ }^{55}$ and further developed in the context of time-resolved diagnostics for plasmas by Geindre et al. ${ }^{56}$. Here we briefly remind the basic principle of the FDI technique. The idea is to split the probe pulse in two pulses delayed by a fixed $\Delta t$. If these two probe pulses are reflected on the sample and sent to a spectrometer, the diffraction grating temporally stretches the 2 pulses allowing them to interfere on the spectrometer detector. We now consider the case when the pump impinges the sample in between the two probe pulses. The complex reflectivity coefficient of the second probe pulse will be then changed by an amount written as $\mathrm{R}=\mathrm{r} e^{i \phi}, r$ being the modulus and $\phi$ the phase of $\mathrm{R}$. The intensity on the spectrometer detector can then be written as:

$$
I(\omega)=I_{0}(\omega)\left(1+r^{2}+2 r \cos (-\omega \Delta t+\phi)\right)
$$

where $\phi$ is the absolute phase, $\omega$ the radial frequency, $I_{0}$ the intensity of each pulse of the probe beam, and $I_{0}(\omega)=\left|\widehat{E}_{0}\left(\omega-\omega_{0}\right)\right|$ where $\widehat{E}_{0}$ is the electric field, $\omega_{0}$ is the central radial frequency of the probe pulse.

In our specific set-up, the measurement for the $\mathrm{S}$ and $\mathrm{P}$ probe polarizations is performed simultaneously. This gives access to the complex dielectric constant of the material and provides a diagnostic of the hydrodynamics of the surface. Measuring $\mathrm{R}_{S}$ and $\mathrm{R}_{P}$, the respective complex reflectivities in $\mathrm{S}$ and $\mathrm{P}$ polarizations can give access to the complex refractive index, $N$, using the following expression:

$$
N^{2}=\left(\frac{1-R_{S}}{1+R_{S}}\right) \cdot\left(\frac{1+R_{P}}{1-R_{P}}\right)
$$

where $\mathrm{N}$ is the complex refractive index expressed as $\mathrm{N}=\mathrm{n}-\mathrm{i} \kappa$. Measuring $\mathrm{R}_{S}$ and $\mathrm{R}_{P}$ allows us to access to $\mathrm{N}^{2}$. The complex dielectric constant during the phase transition, $\epsilon$, can then be deduced from the relation $N^{2}=$ $\epsilon$.

Furthermore, the interesting equality $R_{P}=R_{S}^{2}$ arises by choosing the incident angle of the probe beam on the sample, $\theta$, equal to $45^{\circ}$ and that leads to:

$$
\phi_{P}=2 \cdot \phi_{S} \text { and } r_{P}=r_{S}^{2}
$$

These relations that are independent of the $\mathrm{N}$ index are only valid for probe beams interacting with sharp surfaces, i.e. where the refractive index change happens on a length scale much smaller than the probe wavelength. It is convenient to define the two parameters ${ }^{57}, \beta_{r}$ and $\beta_{\phi}$ as follows:

$$
\beta_{r}=r_{P} / r_{S}^{2}, \beta_{\phi}=\phi_{P}-2 \cdot \phi_{S}
$$

These parameters turn out to be an efficient way to characterize the transition states of the sample. One can distinguish three main cases:

1. $\beta_{r}=1, \beta_{\phi}=0$. This is the canonical case when Fresnel equations are valid: a sharp and immobile interface between vacuum and the sample. This corresponds to an isochoric heating, characterized by a constant volume.

2. $\beta_{r}=1, \beta_{\phi} \neq 0$. The interface is still sharp but moving. The difference in phase results then from the difference in the optical path between the first and the second probe pulse. The amount by which the surface moved, noted $d$, is then related to $\beta_{\phi}$ by the relation:

$$
\beta_{\phi}=\frac{4 \pi}{\lambda} \cos \theta \cdot d
$$

3. $\beta_{r} \neq 1, \beta_{\phi} \neq 0$. A plasma expands toward the vacuum at the sample interface and the second probe pulse interacts with this plasma. If the free electron density, $\mathrm{N}_{e}$, is higher than the critical density $\left(\mathrm{N}_{e}^{c r}=10^{21}(1.06 / \lambda)^{2} \mathrm{~cm}^{-3}\right)$, the beam will be reflected by the plasma surface defined as $\mathrm{N}_{e} \geqslant N_{e}^{c r}$.

\section{B. Experimental set-up}

The FDI set-up, shown in Fig. 9, is made of 3 main blocks: the two stages non-collinear optical parametric amplifier (NOPA) producing the probe pulse, the interaction chamber with the investigated sample and the spectrometer. The Aurore laser beam is firstly split in two: the pump and the probe arms. Each beam is then re-compressed down to $26 \mathrm{fs}$.

The NOPA is built on a well-established scheme shown in Fig. 9. The two stages NOPA can be wavelength tuned between 380 to $680 \mathrm{~nm}$. For practical reasons we choose to run it at $530.89 \pm 0.02 \mathrm{~nm}$. The $1^{\text {st }}$ and $2^{\text {nd }}$ amplification stages deliver pulse energies of nearly 1 $\mu \mathrm{J}$ and $10 \mu \mathrm{J}$ respectively. As the beam goes through several optical components (focusing lens, $\mathrm{SF}_{10}$ rod, 


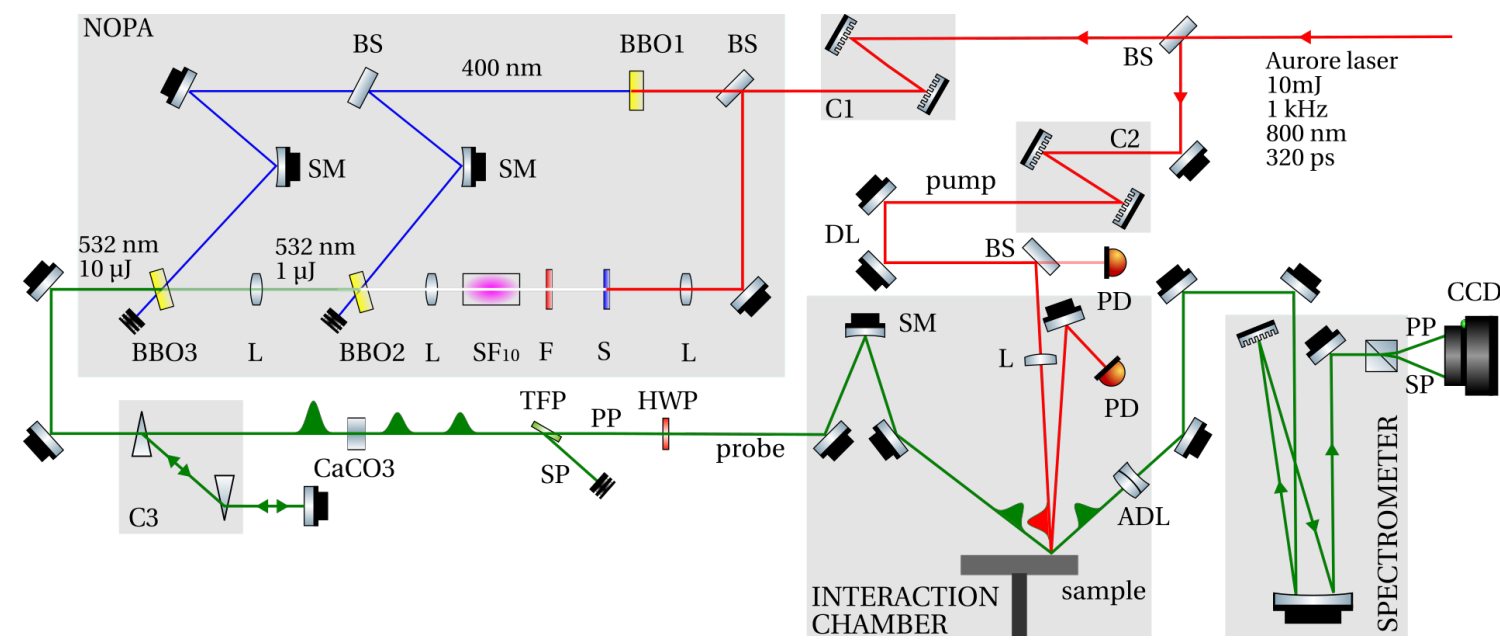

FIG. 9. FDI set-up including the NOPA (BS : beamsplitter, BBO : Beta-Barium Borate crystal, S: sapphire plate, F: 800 nm Notch-filter, SM: spherical mirror, L: Lens, $\mathrm{SF}_{10}$ : heavy-flint glass, $\mathrm{CaCo}_{3}$ : calcite crystal, TFP: thin film polarizer, PP: $\mathrm{P}$ polarized beam, SP: S polarized beam, C1, C2: double pass grating compressor, C3: prisms compressor, PD: photo-diode, ADL: achromatic doublet lens, DL: delay line, HWP: zero order half wave plate)

BBO crystals), the amplified pulse is then recompressed using double-pass configuration in a F2 equilateral prism compressor (C3 in Fig.9). The NOPA centered at $530.89 \pm 0.02 \mathrm{~nm}$ has a FHWM of $11.28 \pm 0.02 \mathrm{~nm}$ corresponding to a Fourier-transform limited duration of $37 \mathrm{fs}$. To measure the pulse duration we have built a wavefront-splitting autocorrelator based on frequency doubling in BBO crystal (60 $\mu m$ thick). The pulse duration (FWHM) is then measured to be $36.9 \pm 1.7 \mathrm{fs}$ from the Gaussian fit.

As stated in Part IV A, FDI requires two delayed probe pulses. That is achieved by inserting a birefringent calcite single crystal. The ordinary and extraordinary refractive indexes that are quite different $\left(\mathrm{n}_{o}=1.66, \mathrm{n}_{e}\right.$ $=1.49$ at $532 \mathrm{~nm}$ ) provide two delayed pulses by $8.5 \mathrm{ps}$ with perpendicular polarizations. This delay fixes the time window of the pump-probe investigation. In order to have both probe pulses with the same polarization on the sample a thin film polarizer is used. The polarization state of the probe beam is then fine tuned by a zero order half waveplate. This is needed to adjust the intensity of the $\mathrm{S}$ and $\mathrm{P}$ reflection on the sample. The beam polarization must not be changed while steered by the different optics. Practically this means that reflection on mirrors must deflect the beam only in one direction (horizontally or vertically) at a time. If this is not the case, $\mathrm{S}$ and $\mathrm{P}$ polarization are mixed, introducing a bias in the interpretation of the data.

The pump and probe beams are then focused onto the sample and overlapped spatially and temporally in the interaction chamber. The pump spot size, which determines the fluence, is measured directly by an imagery set-up based on a 12 bit CCD camera. The $200 \mathrm{~mm}$ plano-convex lens focuses the pump down to $50 \mu \mathrm{m}$ (FWHM) with a quasi perfect Gaussian shape. The $500 \mathrm{~mm}$ focal length mirror of the probe pulses combined with a $0.5 \mathrm{~mm}$ pinhole is aligned to obtain a homogeneous intensity on the sample. The pressure in the chamber can be decreased down to $10^{-5}$ mbar with a turbo-molecular pump. Due to the stability required for such interferometric measurement, no measurement is achieved with running vacuum pumps.

Most of our experiments deals with non-reversible phase transitions, it is then mandatory to refresh the sample in-between two laser pulses. With a $1 \mathrm{kHz}$ laser, the most convenient solution is a rotating target. We developed a specific sample holder which rotates at a speed of 120 RPM. This challenging design is made such that the surface of the sample is always kept in the same plane within $10 \mu \mathrm{m}$. In fact, each data point, i.e. corresponding to a single pump-probe delay, is the average over 1000 laser pulses. If the surface of the sample moves while rotating, this leads to a misalignment on the entrance slit of the spectrometer. To achieve such high accurate positioning over the 100 $\mathrm{mm}$ diameter samples, the sample support is mounted on 3 picomotors which are themselves assembled on the rotating stage. Tuning the picomotors while monitoring the surface with a laser displacement detector (Keyence LKG500, $2 \mu \mathrm{m}$ resolution) allows us to achieve a sub-10 $\mu \mathrm{m}$ displacement. The flatness of the sample itself has to be less than $4 \mu \mathrm{m}$ over the radius.

Finally, the probe beams are steered out of the chamber down to the imaging spectrometer. The interaction point is imaged by an achromatic doublet lenses ( $\mathrm{f}=200 \mathrm{~mm}$ ) on the $200 \mu \mathrm{m}$ entrance slit of the spectrometer. The spectrometer is build on a Fastie-Ebert scheme using a $1 \mathrm{~m}$ focal length spherical mirror and a $2400 \mathrm{l} / \mathrm{mm}$ grating. The $1^{\text {st }}$ order is diffracted back to the spherical mirror so that the slit is re-imaged at the location of the back-illuminated CCD detector (Princeton Instrument Pixis 1024 eXcelon). Slightly before the CCD, the P and $\mathrm{S}$ polarization components of the probe beam are pulled apart by a Wollaston prism. The CCD is a 16 bit cooled camera with pixels size of $13 \mu \mathrm{m}$. Given the dispersion of the grating and the CCD chip size (1024x1024 pixels), the recorded spectral bandwidth is not broader than 2 $\mathrm{nm}$. Such narrow bandwidth also presents the advantage 


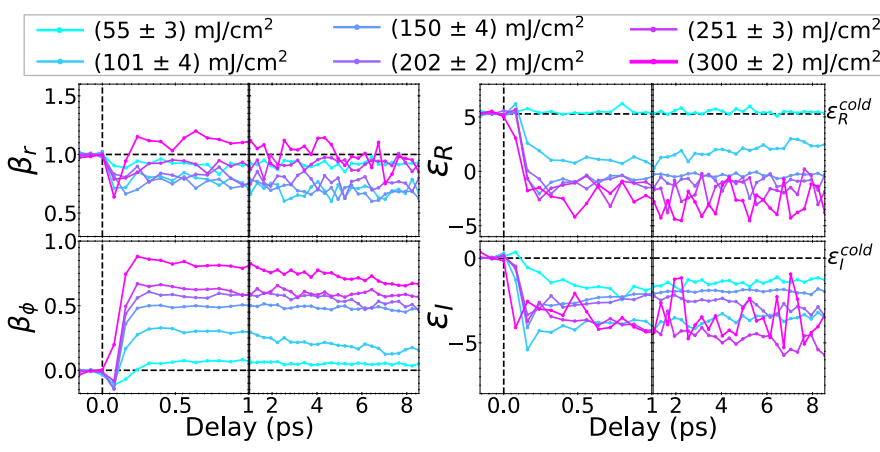

FIG. 10. Left:Time evolution of $\beta_{\phi}$ and $\beta_{r}$ for six different pump intensities. Right: Deduced value of the real (top) and imaginary (bottom) parts of the dielectric constant. The value of the initial $\mathrm{GeS}_{2}$ was measured by ellipsometry to be $\epsilon=5.27+0.04 \mathrm{j}$

to reduce the chromatic aberrations such that the clean wavefront allows to extract properly the $\phi_{S}$ and the $\phi_{P}$ phases of the optical fringes.

\section{Example of results: phase transition in chalcogenide glass}

To illustrate the abilities of the set-up, we present experiment on $\mathrm{GeS}_{2}$ amorphous samples. This material belongs to the chalcogenides, which have several applications in the microelectronic field ${ }^{58}$. Understanding the phase transition dynamics on the femtosecond time scale may help to design better materials. As a consequence numerous studies have been investigating the transition from crystal to amorphous phases ${ }^{59,60}$. For our experiments, the samples were deposited by magnetron sputtering on a Si wafer. The $500 \mathrm{~nm}$ thick layer was then capped with a $10 \mathrm{~nm}$ SiN layer to avoid oxidation. The SiN layer is transparent and do not show any modifications after the irradiation by an intense IR pump pulse.

The main results are shown in Fig. 10. The left graph shows the time dependencies of $\beta_{\phi}$ and $\beta_{r}$. The later is stable around the value of 1 , meaning that the sample is still solid or liquid, and that no plasma has been created even at the highest fluence of $300 \mathrm{~mJ} / \mathrm{cm}^{2}$. Meanwhile, the $\beta_{\phi}$ value increases on the first hundreds femtoseconds and then decreases. This can be directly related to the surface displacement which is first contracting down to $0.75 \mathrm{~nm}$ and then expanding up to $1 \mathrm{~nm}$. The reason of this first contraction is not elucidated yet, but one can infer safely that the expansion on the longer time scale corresponds to the development of a hot liquid phase. As stated previously, $\epsilon_{r}$ and $\epsilon_{i}$ can be deduced from our measurements. The values obtained are corrected from the phase terms due to the hydrodynamics, in fact: $\phi_{\text {measured }}=\phi_{\text {mat }}+\phi_{\text {hydro }}$, where $\phi_{\text {mat }}$ is the phase term due to the material change and $\phi_{\text {hydro }}=\beta_{\phi}$. The resulting $\epsilon$ are shown in Fig. 10. These results needs to be compared to ab-initio simulations at different outof-equilibrium conditions in order to grasp a full under- standing of the phase change are under way.

\section{BEAMLINE 3: TIME-RESOLVED X-RAY PHOTOEMISSION SPECTROSCOPY ON SOLIDS}

As introduced in the paragraph IV, when solids are irradiated with sub-ps laser pulses, specific heating and cooling dynamics of both electronic and atomic populations are expected due to the creation of the out-ofthermodynamical equilibrium state of matter. These dynamics are driven by the material properties, i.e. electron heat capacity, electron thermal conductivity and electron-phonon coupling, which are hardly theoretically described in the non-thermal regime ${ }^{61-64}$, appealing for the development of specific experiments with subpicosecond time-resolved diagnostics ${ }^{65-67}$. One possible way to investigate these properties is to measure the electronic density of occupied states (DOS) that is indirectly driven by the lattice temperature and density ${ }^{68,69}$. A time-resolved measurement of the DOS dynamics therefore brings valuable information on this regime of matter by coupling experiment and modelling. The Aurore beamline 3 has been designed to perform nearIR-pump/XUV-probe time-resolved x-ray photoelectron spectroscopy (TR-XPS) allowing to measure the DOS dynamics on the $\mathrm{ps} / \mathrm{fs}$ timescale. Its principle advantage relies on the achievable spectral range of the probe beam $(\sim 80-100 \mathrm{eV})$ associated with an ultra-short duration $(\sim 26 \mathrm{fs})$. Its brightness and flux stability allow to perform TR-XPS experiments with high reliability.

$\mathrm{X}$-ray photoelectron spectroscopy is a well-established technique in the field of solid state physics. It is based on the photoelectric effect and refers to a spectral measurement of electrons emitted by an X/XUV monochromatic beam of photon energy $h \nu$. The photoelectron kinetic energies $\mathrm{E}_{k}$ are related to their initial binding energies $\mathrm{E}_{B}$ through the relation $\mathrm{E}_{k}=h \nu-\mathrm{E}_{B}-\phi$, where $\phi$ stands for the spectrometer work function ${ }^{70}$. Furthermore, the intensity of the photoelectron current, $\mathrm{I}\left(\mathrm{E}_{k}\right)$, is related to the transition probability from an initial $\Psi_{i}$ to a $\Psi_{f}$ final state, calculated by the Fermi's Golden Rule in the limit of a small perturbation. Hence, if the final state can be approximated by an empty free electron level, the angleintegrated photoelectron signal will be proportional to the sample DOS.

Time-resolved XPS on solid samples, in the frame of phase transition investigation, requires pump intensities in the order of $1.0 \times 10^{15} \mathrm{~W} / \mathrm{cm}^{2}$ depending on the sample thickness. In these conditions, an important photoelectron background, generated by the pump pulse alone, is emitted with kinetic energies up to a few tenths of $\mathrm{eV}$ via multiphoton ionization and Coulomb explosion ${ }^{71}$. This background contribution prevents to use an XUV probe pulse in the 20-50 eV range usually produced in the HHG beamlines. Therefore, we have designed and optimized a HHG-based beamline in the $80-100 \mathrm{eV}$ photon energy range in order to ensure a partial or a complete spectral separation of the XPS pump-probe spectrum (i.e. sample DOS) from the photoelectron background generated by the pump pulse alone. 


\section{A. Experimental set-up: general description}

The TR-XPS beamline is schematized in Fig. 11. The chirped pulse (320 ps) from Aurore laser beam is then split in two parts $(60 \% / 40 \%)$ in order to operate both the HHG probe branch and the near-IR femtosecond pump one respectively. Each arm is independent in terms of pulse duration and energy control thanks to two independent compressors, each equipped with an energy beam attenuator (half-wave plate + polarizing cube).

The pump beam is directly focused by a $2 \mathrm{~m}$ focal length lens onto the sample surface forming an angle of $\sim 3^{\circ}$ with respect to the probe axis. The focused focal spot size of the pump beam is controlled using a beam aperture before the lens, allowing to have an irradiated pump zone on the sample that is nearly 5 times larger than the probe focal spot. Under these conditions, using a focal spot size of $350 \mu \mathrm{m}$ (FWHM), typical fluences of 5. $10^{3} \mathrm{~J} / \mathrm{m}^{2}$ can be reached. The pump/probe relative delay is controlled through a translation stage installed on the pump optical path. The probe beam is steered into the vacuum set-up right after compression in order to produce XUV photons by HHG. As highlighted in Fig. 11, this HHG beamline branch can be divided in three parts: (1) generation, (2) monochromatization and focusing, and (3) characterization.

The first part consists in the Aurore laser beam focused in a variable-length gas cell using a spherical mirror of $1 \mathrm{~m}$ focal length. The XUV generation is optimized in the range of $80-100 \mathrm{eV}$ photon energies using neon as generating gas. Typically, gas pressure in the cell is around 200 mbar. A differential pumping stage is installed at the output of the generation cell chamber in order to reduce the surrounding pressure from $10^{-2}$ to $10^{-5}$ that is necessary to avoid gas absorption of the XUV radiation and to reach UHV conditions required in the XPS interaction chamber.

The HHG selection is achieved through a pair of multilayer mirrors (ML) (OptiX fab GmbH, Jena, Germany) based monochromator. This choice, compared to a grating-based monochromator, has been made in order to avoid the pulse stretching and preserve the nominal duration of the high-order harmonics driven by the 26 fs IR pulses. For that purpose, the MLs have been designed to present a single peak of reflectivity in the spectral range of interest $(\sim 80-100 \mathrm{eV})$ where the central energy is a function of the incidence angle. As such, changing the angle of incidence on the MLs allows us to select a single harmonic. In addition, the second ML is mounted on a translation stage to keep constant the output axis for any incident angles (all selected harmonics). These motorizations are remotely controlled through a LabVIEW program that adjusts the two MLs angles of incidence and $2^{\text {nd }}$ ML translation position. The residual laser radiation is filtered out using a $110 \mathrm{~nm}$ thick $\mathrm{Zr}$ film (Luxel Corp.) with a transmission of $0.52 \pm 0.06$ (measured for $\mathrm{H} 55,85.27$ $\mathrm{eV}$ ), after the monochromator. The selected harmonic beam is then focused onto the sample surface in the
UHV interaction chamber using a $1 \mathrm{~m}$ grazing incidence gold-coated toroidal mirror. These monochromatizating and focusing parts of the probe branch are installed in independent vacuum chambers where the pressure reaches $10^{-7}$ mbar and $10^{-8}$ mbar respectively.

The HHG beamline ends with a characterization stage, including a retractable avalanche photodiode and an XUV spectrometer. The latter is composed of an XUV diffraction grating and a microchannel plate/phosphor screen detector system that is coupled with a CCD camera (12 bit). It is located after the interaction chamber in a dedicated vacuum set-up. This final HHG characterization is required for the XUV photon flux daily optimization that is highly sensitive on the laser beam characteristics and gas cell parameters.

The probe and pump beams are recombined in the so-called interaction chamber where UHV conditions are reached $\left(10^{-9}\right.$ mbar $)$. It is equipped with all typical instruments required for sample preparation and XPS experiments: a $(\mathrm{x}, \mathrm{y}, \mathrm{z}, \Theta)$ manual sample holder, cooling $\left(-70^{\circ} \mathrm{C}\right.$ ) and annealing (up to $370^{\circ} \mathrm{C}$ ) sample systems, an Ar ion sputtering gun for sample surface cleaning, an electron gun for charge compensation when working with low conductivity materials and a classic twin anode $\mathrm{Al} / \mathrm{Mg} \mathrm{X}$-ray source for sample surface chemical characterization by XPS (RS40B1 from Prevac). Finally, XPS and TR-XPS electron spectra are recorded at an angle of $45^{\circ}$ (with respect to the laser axis) with an Electron Energy Analyzer (Thermo VGScientific Clam IV) with a 9 channeltrons detection head.

\section{B. HHG beamline characterization}

As mentioned above, the highly non-linear HHG mechanism is sensitive to numerous parameters of the driving laser pulse (duration, temporal chirp, spatial profile of the focused beam, intensity and focusing configuration) and of the gas cell (interaction length, pressure and gas nature). In addition, photoionization cross sections are low enough to forbid single pulse acquisition and require long accumulation time. For those reasons, the HHG generation must be precisely controlled and adjusted in order to ensure an optimal quality and intensity. It also requires a stable monochromatized photon flux and a focusing diameter smaller that the pump spot. Therefore, the beamline optimization has been performed during a dedicated experimental commissioning which resulted in the following technical choices.

The precise optimization of the HHG has been made possible mainly through a home-made gas cell design adjustable in length from 1 to $20 \mathrm{~mm}$. The maximum XUV photon flux has been obtained in the range of 3 to 12 $\mathrm{mm}$ length of the cell with a corresponding gas pressure around 100 to 200 mbar. The XUV photons flux stability has been evaluated to be around $8 \%$ during 10 operating hours. It is mainly driven by: (1) a remote pressure stabilizer; (2) a heat-dissipating adjustable diaphragm located before the spherical mirror used to control the laser beam focusing conditions (intensity, focal 


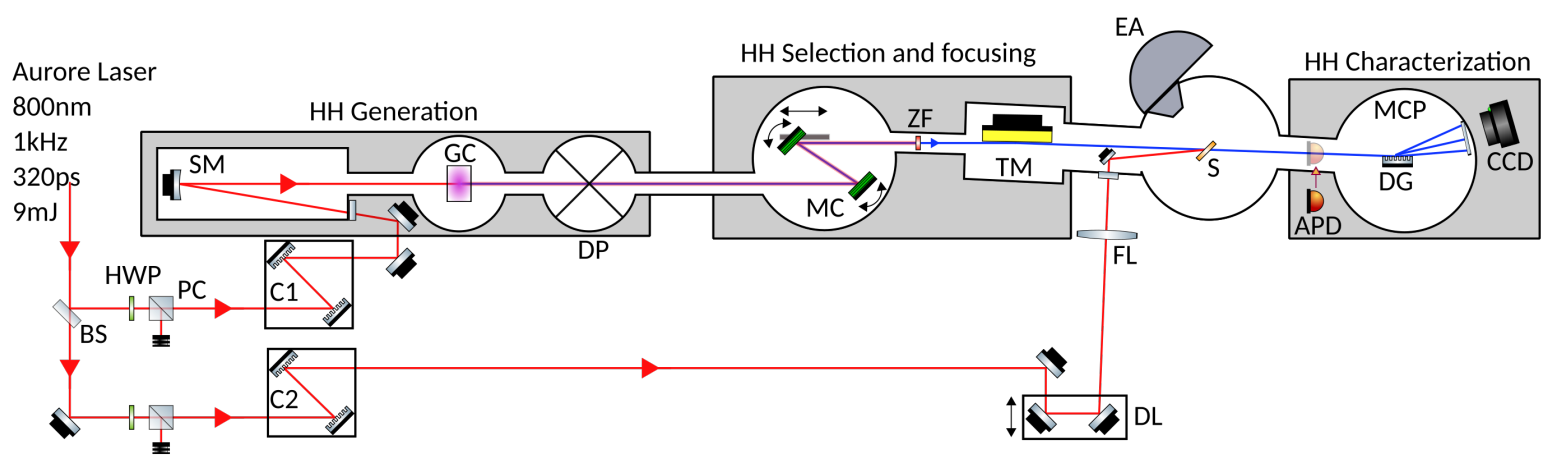

FIG. 11. TR-XPS beamline set-up (BS: beamsplitter, HWP: half-wave plate, PC: polarizing cube, C1(2): pump (probe) compressor, SM: spherical mirror, DL: delay line, FL: focusing lens, GC: gas cell, DP: differential pumping, ZF: zirconium filter, MC: monochromator, TM: toroidal mirror, EA: electron energy analyzer, S: sample, APD: avalanche photodiode, DG: XUV diffraction grating, MCP: microchannel plate, CCD camera).

spot size and Rayleigh length); (3) pre-drilled cell walls made of $200 \mu \mathrm{m}$ thick brass foils. An upper value of $85 \times$ $60 \mu \mathrm{m}^{2}$ was found for the XUV focal spot diameter on the sample plane. It has been estimated by imaging the residual near-IR beam impinging on sample surface. One should underline that this residual beam is also useful to determine the pump-probe 0 fs delay by imaging the appearance of an interference pattern on the sample surface while both beam are synchronized.

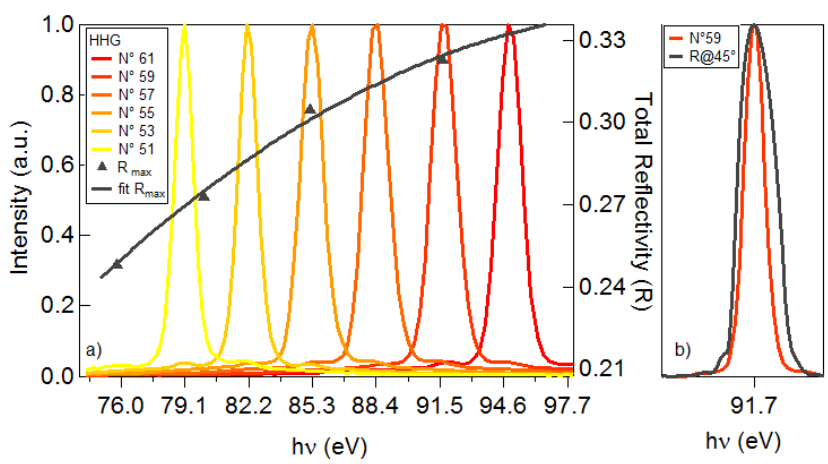

FIG. 12. a) Single harmonic selection with wavelength dependence of the absolute reflectivity of the monochromator. b) Spectral bandwidth of the XUV monochromator at $45^{\circ}$ incidence angle and spectral selection of H59.

The spectral selection of one harmonic, illustrated in Fig. 12, is achieved by the ML monochromator described above. The monochromator reflectivity is centered at an adjustable photon energy depending on the incidence angle and yields a total measured peak reflectivity ranging from $20 \%$ to $32 \%$ depending on the selected order of the harmonic. The spectral bandwidth of the XUV monochromator ranges from $2.25 \mathrm{eV}$ to 2.37 $\mathrm{eV}$ (FWHM), which is larger than the natural spectral width of generated high-order harmonics reported in Table 1. Therefore and as we experimentally confirmed, the monochromator only selects a single harmonic (see Fig. 12) with no further monochromatization, ensuring a maximization of the XUV photon flux on the sample surface. The overall spectral resolution of HHG beamline is thus given by the high-order harmonic bandwidth ranging from 0.91 to $1.23 \mathrm{eV}$ (FWHM).

The XUV photon flux impinging on the sample surface has been optimized by a precise adjustment of the above mentioned parameters. It has been measured using a calibrated APD photodiode ${ }^{72}$ (XUV-100 from OSI Optoelectronics) located at the interaction chamber exit for a selected photon energy range from H51 to H61. The XUV photon flux $\mathrm{N}_{X U V}$ ranges from 0.21 to $2.59 \times 10^{7}$ photons $/ \mathrm{s}$ at $1 \mathrm{kHz}$ repetition rate. The corresponding values for each harmonic are reported in Table III.

\begin{tabular}{c|c|c|c}
$\begin{array}{c}\text { Harmonic } \\
\text { order }\end{array}$ & $\begin{array}{c}\text { Central energy } \\
\mathrm{eV}\end{array}$ & $\begin{array}{c}\mathrm{N}_{X U V} \\
10^{7} \text { photons/s }\end{array}$ & $\begin{array}{c}\text { Bandwidth } \\
\mathrm{eV} \text { (FWHM) }\end{array}$ \\
\hline 51 & 79.00 & $1.20 \pm 0.30$ & $0.91 \pm 0.17$ \\
53 & 82.15 & $2.05 \pm 0.30$ & $0.94 \pm 0.18$ \\
55 & 85.27 & $2.59 \pm 0.26$ & $1.13 \pm 0.22$ \\
57 & 88.40 & $1.95 \pm 0.24$ & $1.20 \pm 0.25$ \\
59 & 91.69 & $0.76 \pm 0.22$ & $1.23 \pm 0.26$ \\
61 & 94.50 & $0.21 \pm 0.20$ & $1.17 \pm 0.26$
\end{tabular}

TABLE III. Characteristics of XUV beamline after spectral selection of a single generated harmonic at the sample location.

\section{Example of results: space charge effect in TR-XPS experiments}

In order to illustrate the beamline capabilities, we present the measurements of probe XPS spectra (Fig. 13) recorded on two different samples: $1 \mathrm{~mm}$ thick $\mathrm{Au}$ and $\mathrm{Cu}$ polished (roughness $<0.01 \mu \mathrm{m}$ ) commercial single crystals with a (111) surface orientation (Mateck), and pump/probe (Fig. 14) XPS spectra recorded on the Au sample. This preliminary experimental campaign has been performed to test the new designed HHG beamline performance and to investigate the expected so-called space charge effect ${ }^{73-75}$ occurring in pulsed XPS experiments. 
The samples were cleaned applying a procedure of consecutive sputtering and annealing cycles. Their respective PES spectra were recorded in the valence band energy range corresponding to $5 \mathrm{~d} / 6 \mathrm{~s}$ and $3 \mathrm{~d} / 4 \mathrm{~s}$ bands of $\mathrm{Au}$ and $\mathrm{Cu}$ samples respectively. Using a $20 \mathrm{eV}$ pass energy on the electron analyzer and the H53 $(82.15 \mathrm{eV})$, the overall spectral resolution is estimated to be around 1.54 $\mathrm{eV}$ (FWHM). The resulting spectra, reported in Fig. 13 have been acquired in 7 minutes each.
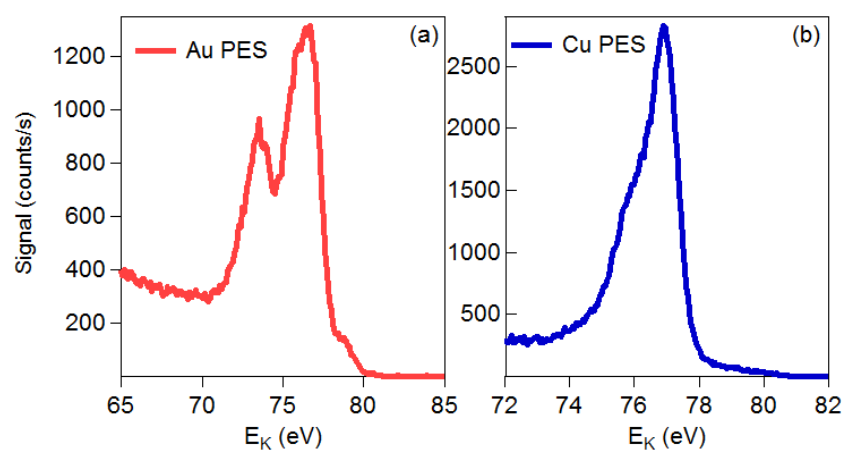

FIG. 13. Probe measurements: PES spectra from (a) Au and (b) $\mathrm{Cu}$ single crystals recorded using the harmonic H53.

In TR-XPS experiments, the space charge effect results from the electrons generated by the high intensity pump pulse. The high density electron cloud ejected by the pump pulse influences the central peak energy and the structure of the probe electron spectrum (i.e. the valence band in our case) via Coulomb interactions while traveling in vacuum towards the detector. This inherent drawback of TR-XPS experiments has thus been extensively studied in various contributions ${ }^{76-78}$ aiming at its minimization/suppression. It can also be addressed using the presented instrument.

In Fig. 14, we present a set of measurements of the $\mathrm{Au}$ XPS spectra recorded at different pump/probe delays illustrating the space charge-induced modifications triggered by the pump near-IR laser pulse. At negative delay, photoelectron emitted by the XUV pulse are energy shifted by the IR- pulse. As clearly evidenced here, the delay-dependent changes with a maximum at zero delay time not only shifts the Au valence band spectrum but also induces an important broadening and distortion of the initial energy distribution of photoelectron. It is of prime importance to reduce this effect in order to disentangle this artifact from the investigated physical phenomenon such as an ultrafast phase transition. This part of the study is under development and will be the subject of a future dedicated publication.

\section{BEAMLINE 4: TIME-RESOLVED PHOTO-LUMINESCENCE SPECTROSCOPY}

The AURORE beamline 4 is dedicated to luminescence measurements in condensed matter. The luminescence of dielectrics, wide gap semi-conductors crystals and nano-particles solids is studied to obtain new information on the dynamics of excitons and

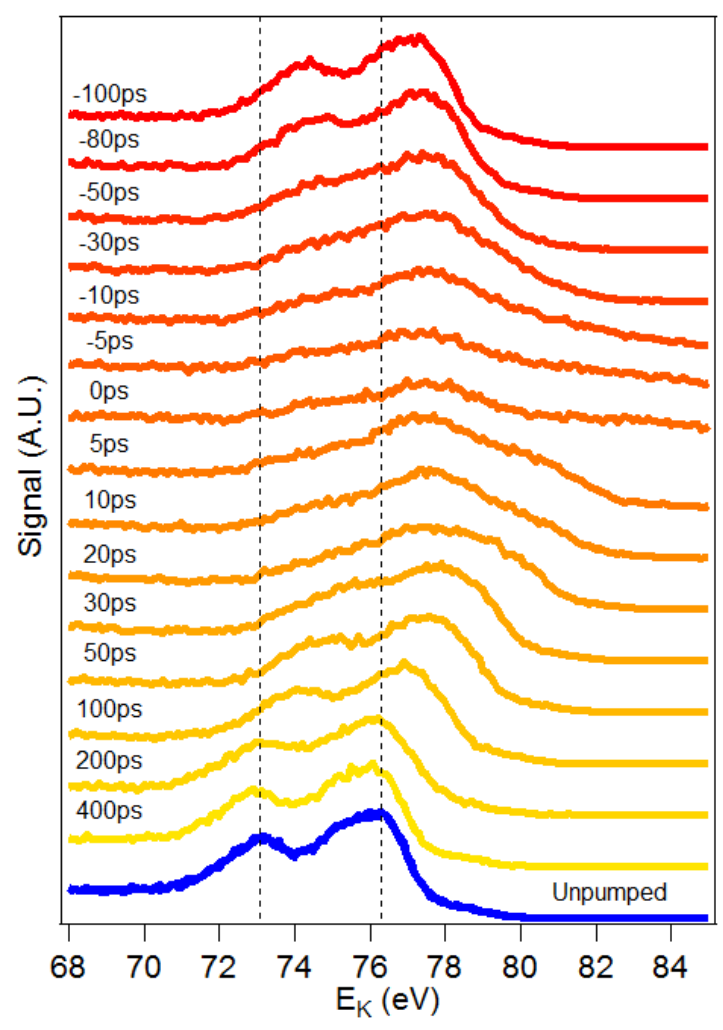

FIG. 14. Pump-Probe measurements: PES spectra from Au single crystal as a function of the pump/probe delay. A vertical shift is applied for clarity reasons. Pump: $\tau=1 \mathrm{ps}$, $\mathrm{F}=655 \mathrm{~J} / \mathrm{m}^{2}$, Polar $\mathrm{P}$ and $\Theta=45^{\circ}$. Probe: Harmonic H53 $(82.15 \mathrm{eV}), \tau=26 \mathrm{fs}$. As a reference, the probe-only spectrum (unpumped sample) is reported in blue. The dashed lines indicate the $5 \mathrm{~d}$ doublet peak positions of the unpumped sample. The delay 0 ps corresponds to the time overlap of pump and probe pulses while the negative delays are related to sample probing before the pump pulse irradiation.

electron relaxation. Depending on different experimental parameters (temperature, excitation energy, excitation density,...), the luminescence emissions are spectrally and temporally resolved in order to reveal the fundamental processes which govern the energy relaxation in solids. The experimental studies on condensed matter need photons pulses in the VUV domain (wavelength selection of a single harmonic) and the IR-UV range (up to $6.2 \mathrm{eV}$ ). As it is shown on figure 15 , this beamline is well adapted for these measurements.

\section{A. Experimental set-up: general description}

This beamline and the experimental set-up are schematized in Fig. 15. The $800 \mathrm{~nm}, 26 \mathrm{fs}, 1 \mathrm{kHz}$ laser beam ( $<9 \mathrm{~mJ} /$ pulse) can be split and used to generate high-order harmonics pulses and/or first harmonics (400 $\mathrm{nm}, 266 \mathrm{~nm}, 200 \mathrm{~nm}$ ). High-order harmonic generation (HHG) is performed by the interaction of focused 800 $\mathrm{nm}$ laser pulses with a rare gas in an interaction cell (GC). The output VUV and laser beams are steered into 
a VUV monochromator without entrance slit. A plane mirror (DM) removes the zero order of the grating out of the beam line by adjusting its translation position in function of the incident angle. The combination of a plane XUV grating (DG) (470 lines $/ \mathrm{mm}$ ) and a toroidal gold mirror (TM) allows to focus and select through an exit slit (ES) one single VUV harmonic. The wavelength selected VUV radiation is then focused in the experimental chamber by a second toroidal gold mirror (TM). In these conditions, depending on the nature of the generating gas, the available VUV radiation covers the spectral range from $10 \mathrm{~nm}$ (H79) to $73 \mathrm{~nm}$ (H11). On the sample, the VUV beam size is around $250 \mu \mathrm{m}$ with $10^{4}-10^{5}$ photons/pulse in one harmonic at $35 \mathrm{~nm}$ (H23 in argon). Due to the temporal stretching of the the monochromator (pulse-front tilt induced by the diffraction grating), the VUV pulse duration on the sample increases to reach $500-600$ fs. The sample is placed in the vacuum chamber $\left(10^{-8}\right.$ mbar $)$ where it can be translated in the $\mathrm{x}, \mathrm{y}$, and $\mathrm{z}$ directions and cooled from room temperature to around $13 \mathrm{~K}$ by a liquid helium cryostat. When using the fundamental, the second, third or fourth harmonics of the laser pulses to excite the sample, the beam is directly transported to the experimental chamber via a table top set-up with BBO crystals able to generate the frequency up-conversion to HG-2,3,4. Two $\mathrm{MgF}_{2}$ prisms select the wavelength. The IR-UV beam can be focused on the sample by a lens mounted on a translation stage to monitor the spot size (Z-scan measurements). The photo-luminescence (PL) is collected by a lens and steered to a spectrometer through an optical fiber. The time-integrated PL spectrum is measured with a spectrometer (TRIAX Jobin-Yvon 190) equipped with 3 gratings (1200 lines/mm and 300 lines/mm high and low range) and a charged-couple device (ANDOR iCCD) camera. This spectrometer can also be used as monochromator with a photomultiplier (MCP-Hamamatsu R3809U-58) working in the photoncounting mode to record the PL kinetics decay with 50 ps time resolution (FWHM). In order to access to shorter time resolution, in the sub-ps range, a set-up of frequency mixing (FM) between the luminescence emission and the $800 \mathrm{~nm}$ laser pulse has been developed with time resolution down to $400 \mathrm{fs}$.

\section{B. Example of results}

We performed experiments on $\mathrm{ZnO}$ crystal sample as presented in Fig. 16. Numerous studies have been investigating about this wide band gap semi-conductor and controversial results still remain, particularly about the dynamics properties of the DX bounded exciton. In figure 16 , the radiative decay of the DX exciton luminescence measured at $369 \mathrm{~nm}$ wavelength emission are shown at $14 \mathrm{~K}$ for three excitation wavelengths. In red color, the crystal was excited by 26 fs pulses of $800 \mathrm{~nm}$ photons (focused beam with $2 \mu \mathrm{J} /$ pulse) leading to threephotons absorption process to promote an electron from the top of the valence band to the conduction band. In blue color, the excitation photon energy is $4.7 \mathrm{eV}$ (third harmonic of $800 \mathrm{~nm}, 2 \mathrm{~mm}$ spot size diameter with 5 $\mathrm{nJ} /$ pulse) and in green color, the $\mathrm{ZnO}$ crystal excitation is triggered by VUV pulses at $48 \mathrm{eV}\left(\mathrm{H} 31, \sim 10^{4}\right.$ photons/pulse). Excitation by UV and VUV photons leads to a direct transition (one absorption photon) from valence band to conduction band. These PL transients occur with very different decay times $\left(\tau_{l}\right)$ through which information about the DX formation primary processes and dynamics depending on its environment can be obtained.

It is also well known that density of excitations controls the process of energy transfer, localization of excitations, scintillation yield and decay, storage of energy, etc. At high excitation densities, above $10^{17} \mathrm{~cm}^{-3}$, one usually observes effects of interaction between electronic excitations and could result not only in quenching of emission centers (excitons), but also can stimulate different processes of creation of new excited states with super-linear dependence of excitation density.

Time-resolved luminescence Z-scan technique (translation of the focusing lens along the axis of the laser beam) gives the opportunity to obtain consistent data for dependence of the luminescence yield, spectrum and decay kinetics on excitation density. Figure 17 shows the variation of the excitonic luminescence of $\mathrm{CsPbCl}_{3}$ crystal at $200 \mathrm{~K}$ excited by $266 \mathrm{~nm}$ photons (50 nJ/pulse) during the Z-scan. "The insert gives the maximal density of excitations obtained at different lens positions. The calculations of the created excitations density at different lens positions can be found in reference ${ }^{79}$.

\section{BEAMLINE 5: PHOTOCHEMISTRY SPECTROSCOPY ON SMALL QUANTUM SYSTEMS}

The Aurore beamline 5 is dedicated to investigation of relaxation dynamics in small quantum systems: molecules and nano-particles. The long-term stability of the laser pointing and intensity of Aurore allow to record static ${ }^{80}$ or time-resolved photoelectron ${ }^{8,43,81-84}$ or ion imaging ${ }^{85-87}$ for more than 12 hours without any realignment.

\section{A. Experimental set-up}

The experimental set-up consists of a molecular beam chamber, coupled to a velocity-map imaging (VMI) spectrometer by a long-neck or short-neck skimmer with diameters of 0.7 to $1.5 \mathrm{~mm}$. One particularity of this set-up is the pumping capacity of $3200 \mathrm{l} / \mathrm{s}$, that allows to use different molecular beams depending on the sample:

- a 1-kHz pulsed Even-Lavie valve ${ }^{88}$, synchronized with optical pulses from the Aurore laser. The molecules of interest can be inserted close to the nozzle in a large cartridge container and the temperature can reach up to $250^{\circ} \mathrm{C}$. The typical consumption for this valve when running at $1 \mathrm{kHz}$ repetition rate, is $\sim 3 \mathrm{~mL} /$ day. The $350 \mathrm{~mm}^{3}$ cartridge 


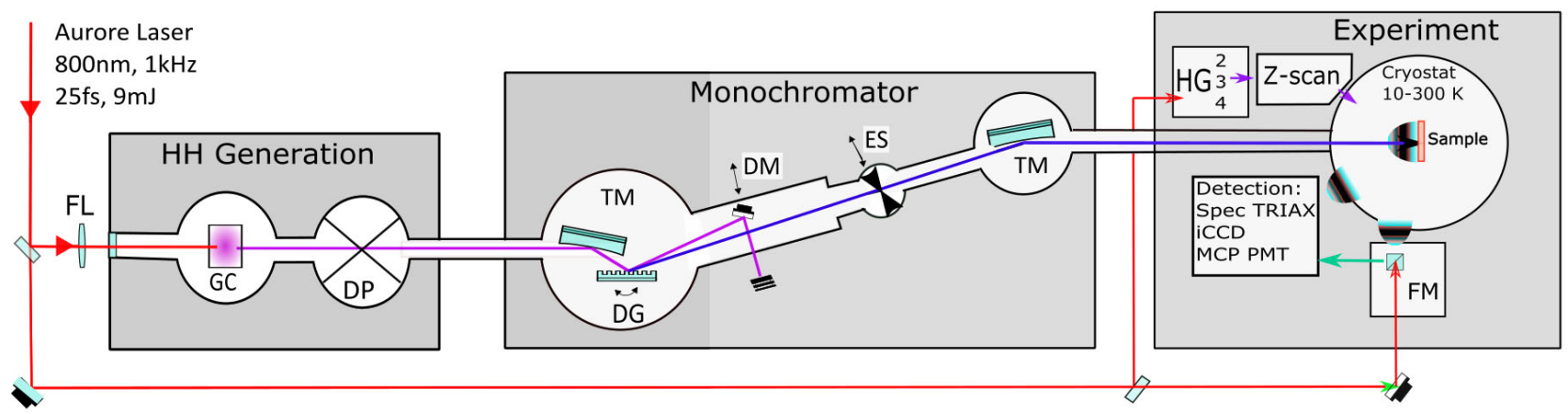

FIG. 15. AURORE VUV-beamline 4 and luminescence experimental set-up: FL focusing lens, GC: gas cell, TM: toroidal mirror, DG: diffraction grating, DM: deviation mirror, ES: exit slit, TM: toroidal mirror, FM: frequency mixing set up, HG-2,3,4: 2nd, $3^{\text {rd }}$ and $4^{\text {th }}$ harmonic generation set-up.

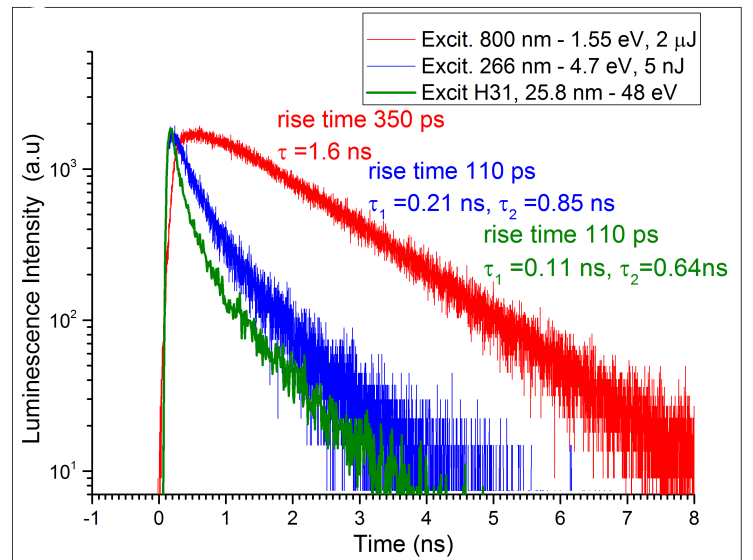

FIG. 16. Luminescence decay measured at $369 \mathrm{~nm}$ wavelength emission of the DX exciton in $\mathrm{ZnO}$ crystal at 14K: Wavelength excitation at $800 \mathrm{~nm}$ (red), $266 \mathrm{~nm}$ (blue) and VUV $25.8 \mathrm{~nm}$ (green) femtosecond pulses. The characteristic rise times and decay times are given for each case.

volume is in general large enough to run the experiment for two consecutive weeks. The molecular beam is generally seeded with high pressure helium (above 7 bars), which enables efficient cooling in the supersonic expansion, leaving the molecules in the low rotational and vibrational levels of the electronic ground state. Depending on the diameter and shape of the nozzle (trumpet/conical), Van der Waals complexes can be also formed in the expansion, in particular when $\mathrm{N}_{2}$ or Ar argon are used as seed gas.

- a $100 \mu \mathrm{m}$ nozzle coupled to a bubbler tank with liquid sample. This creates an effusive molecular beam. In the example shown further, the liquid samples were made of NHS or NHS-C-Dots with typical concentration of $5 \mathrm{~g} / \mathrm{L}$ and $5.8 \mathrm{~g} / \mathrm{L}$, respectively. There is no heating element in this continuous molecular source, which is running using $\mathrm{N}_{2}$ as seeding gas at a typical pressure of 600 mbar. This allows for aerosolisation without decomposition. As there is no drying stage in the system, the

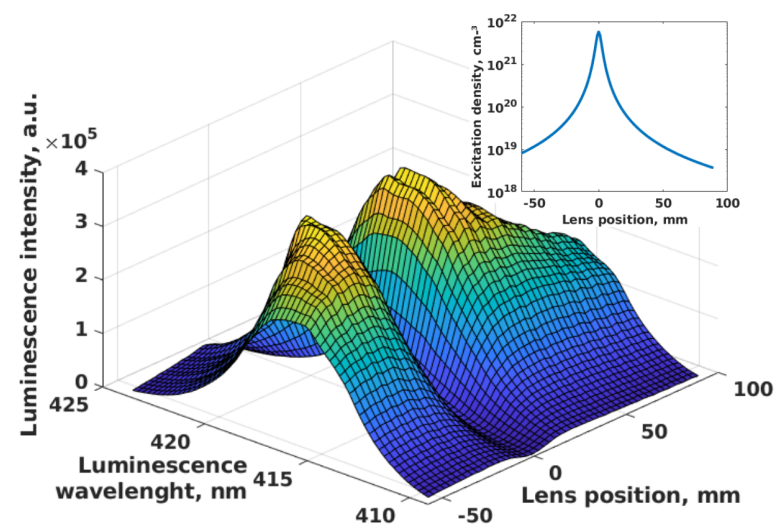

FIG. 17. Variation of the excitonic luminescence of $\mathrm{CsPbCl}_{3}$ crystal excited by $266 \mathrm{~nm}$ photons (50 nJ/pulse) during Zscan. Insert: dependence of the maximal density of excitations near the surface at the spot center.

bubbler creates a wet aerosol of the aqueous sample and the carrier gas.

- the set-up of an aerodynamical lens is currently under construction. This system will provide a collimated jet of nano-particles as the ones routinely used in synchrotron facilities ${ }^{89,90}$.

- a simple oven that can be heated up to $\sim 500^{\circ} \mathrm{C}$ with a set of different nozzle diameters.

The nozzle-skimmer distance can be varied with a minimum distance nozzle-to-VMI center of $120 \mathrm{~mm}$.

From the optical point of view, tunable pump or probe pulses can be produced from $750 \mathrm{~nm}$ down to $200 \mathrm{~nm}$ in a three-stages NOPA. The other probe/pump pulse is simply one of the harmonics (first to fourth) of $800 \mathrm{~nm} \mathrm{Au}-$ rore laser beam. Note that the polarization states of the pump and probe pulses can be fully controlled (variable $\mathrm{S}_{3}$ Stokes parameter) over the whole UV-IR bandwidth and fully characterized by polarimetry. This is crucial for time-resolved circular dichroism experiments ${ }^{8,43}$.

Following photoionization, the ionic fragments or photoelectrons are extracted in a direction perpendicular to the plane defined by the laser and the molecular beam, and 
detected at the end of a $40 \mathrm{~cm}$ time-of-flight (TOF) tube using an imaging detector ( $7 \mathrm{~cm}$ in diameter) consisting of two micro-channel plates (MCP) coupled to a phosphor screen and a CMOS camera. The electrostatic lens is magnetically protected by a Mu-metal tube. To obtain sufficient mass resolution, while retaining a short TOF to avoid dissociation during the flight time and as well to improve the detection efficiency on the MCP, a $4 \mathrm{kV}$ voltage is applied to the repeller plate of the VMI. The preamplified signal (factor 200) extracted directly from the phosphor screen by a $100 \mathrm{pF}$ capacitor is recorded to capture the TOF mass spectrum of the species produced by photoionization. To illustrate the potential of this beamline, we will present two experiments: mass-spectrometry of complex systems as carbon dots (C-Dots) as well as femtosecond pump-probe spectroscopy of $\mathrm{CH}_{3} \mathrm{I}$ predissociation by REMPI- velocity map ion imaging.

\section{B. Example of mass Spectroscopy of Carbon Nanodots and their Building Block N-hydroxysuccinimide (NHS)}

The characterization of the surface ligands of carbon nanodots is of outmost importance for the understanding of the photo-luminescence of C-Dots. We therefore used ultraviolet (UV) and infrared (IR) fs laser pulses to induce photoionization and detachment in freestanding NHS- C-Dots ${ }^{91}$. The obtained photofragment mass spectra were compared to the fragmentation of NHS $\left(\mathrm{C}_{4} \mathrm{H}_{5} \mathrm{NO}_{3}, 115 \mathrm{amu}\right)$ aqueous solution at the same excitation energies. These NHS give us important reference data for the study of C-Dots and allows us to disentangle purely molecular processes from solvated or quantum dot induced processes. More specifically, one-color femtosecond multiphoton ionization was carried out at 270 $\mathrm{nm}(4.6 \mathrm{eV})$ and $800 \mathrm{~nm}(1.55 \mathrm{eV})$ wavelengths for the free-standing C-Dots and NHS (starting from the aqueous solution). A NIR fs laser multiphoton ionization can be in general compared to a very soft electron ionization, while UV-fs laser radiation favors fragmentation before multiphoton ionization. The UV fs-induced ionization is known to provide useful structural information on large molecular systems due to the saturation of the ionization rate that provides an equal efficiency for the different compounds. ${ }^{92}$

The third-harmonic pulse (THG) at $270 \mathrm{~nm}$ with a typical bandwidth of $2 \mathrm{~nm}-33 \mathrm{meV}$ (Fourier limited duration of $55 \mathrm{fs}$ but measured at $90 \mathrm{fs}$ ) is used for multiphoton ionization at intensities around $10^{13} \mathrm{~W} / \mathrm{cm}^{2}(30 \mu \mathrm{J} /$ pulse focused by a $250 \mathrm{~mm}$ thin lens). The fundamental pulse at $800 \mathrm{~nm}$ has a typical pulse duration of 26 fs and is focused with a $300 \mathrm{~mm}$ thin lens to reach intensities around $10^{14} \mathrm{~W} / \mathrm{cm}^{2}(140 \mu \mathrm{J} /$ pulse $)$. In the fs regime, these IR and UV intensities allow us to reach the saturation of ionization. ${ }^{92}$ The polarization of the UV and IR pulses were linear and set parallel to the detector plane.

Each mass spectrum shown here was recorded over $10^{4}$ laser pulses by a $4 \mathrm{GHz}$ oscilloscope. The mass calibration was done with xenon.

The femtosecond photoionization mass spectrum at 800 nm shown in Fig.18 displays a moderate degree of fragmentation compared to the one recorded at $270 \mathrm{~nm}$ exci- tation wavelength. The $18 \mathrm{amu}$ photoionization of water (IP=12.62 eV-NIST value) is significant, implying that the $13 \mathrm{eV}$ range is energetically reached with NIR. The $18 \mathrm{amu}$ peak is stronger at $270 \mathrm{~nm}$ due to Rydberg resonances encountered around $9 \mathrm{eV}$ in water. This peak was even saturating the detector for NHS-water sample. We stress the fact that ionized C-Dots are too heavy to fly the $40 \mathrm{~cm}$ TOF and only its ligands or fragments can be detected with the heaviest masses observed (not shown here) at 130, 145 and 149 amu. In both samples, protonated NHS at $116 \mathrm{amu}$ is revealed by the soft ionization at $800 \mathrm{~nm}$. At $270 \mathrm{~nm}$ multiphoton ionization, the NHS peak with or without a hydrogen loss is more important in the C-Dots sample compared to the NHS bare ones. At $800 \mathrm{~nm}$ excitation wavelength the dominant peak of the mass spectra is the $58 \mathrm{amu}$ that is not seen in the bare NHS ionization ${ }^{89}$. It corresponds to a $[\mathrm{ONCO}]^{+}$radical fragment of NHS. The fragment $\left[\mathrm{C}_{2} \mathrm{H}_{3} \mathrm{NOH}\right]^{+}$would also give a signal at $58 \mathrm{amu}$, but its formation requires both proton transfer and cleavage of the $\mathrm{CO}$ or NO bonds, which is considered less likely. The relative intensity of the $[\mathrm{ONCO}]^{+}$fragment is higher in C-dots than in NHSwater sample, which may be an indication of the ring opening during the synthesis of the C-dots. In a water environment, once the NHS chromophore photodissociates, the solvation is in play to form more stable species with paired electrons. As seen on Fig. 18, such efficient rearrangement are involved to produce the strong signals at 76 and $94 \mathrm{amu}$ corresponding to $\left[\mathrm{ONCO}\left(\mathrm{H}_{2} \mathrm{O}\right)_{n=1,2}\right]^{+}$. This same progression is also observed upon ionization at $270 \mathrm{~nm}$, but with a double peak structure corresponding to one proton mass difference between the peaks. These peaks are greatly enhanced using $270-\mathrm{nm}$ ionization, especially in the C-Dots sample. Finally, the main peak that appears on all mass spectra is at $43 \mathrm{amu}$, which can be an acetyl group $\left(\mathrm{CH}_{3} \mathrm{CO}\right)$, formed after a proton transfer within a pristine NHS molecule, or $\mathrm{CNOH}$, with hydroxyl group bound either in $\mathrm{N}$ or $\mathrm{C}$, which requires a proton transfer as well. Such strong rearrangement processes can easily be at play during the dissociation that follows ionization. This type of rearrangement explains as well the presence of methyl (peak at $15 \mathrm{amu}$ ) or either the proton progressions built on $29 \mathrm{amu}(\mathrm{COH})$ and 28 amu $\left(\mathrm{C}_{2} \mathrm{H}_{2}\right)$. It is quite surprising to not detect larger carbon species in the $\mathrm{C}$-Dots sample as expected from the graphitic planes observed on the TEM images ${ }^{89}$. We discard the possibility that our carrier gas transportation from the bubbler to the nozzle is not efficient enough to provide a significant proportion of C-Dots in the laser interaction zone. Indeed, with similar pressure and distances, we have been able to record photoelectron spectra from the same C-Dots sample in the soft X-ray regime ${ }^{89}$. This absence of large carbon fragments and the weak proportion of $\mathrm{C}_{3} \mathrm{X}$ is certainly due to the large proportion of surface ligands as well as water solvation that both prevent fragmentation of the carbon core of the C-Dots as concluded by depth profiling using X-ray Photoelectron Spectroscopy (XPS) ${ }^{89}$.

As a conclusion, whatever the ionization regime, the mass spectra obtained from the two samples are quite similar at lower mass $(<58 \mathrm{amu})$. However, for the higher masses, the strong differences as a function of the ioniza- 
tion regime (UV or IR) allows to appreciate the significant solvation and clusterization that can easily proceed from NHS core. The similarities between the initially aqueous samples, NHS/C-Dots and NHS, indicate that their surfaces- from where the fragments originate- are rather similar. An especially important observation is the presence of the protonated parent ion in both samples, suggesting that the graphitic core is effectively covered with NHS molecules, i.e. the free-standing NHS-C-Dots aerosol prepared from aqueous solution does not seem to contain only parts or fragments of NHS molecule, but the bare molecules as well. However, we have to emphasize that this method studies the whole aerosol produced by bubbling nitrogen gas through aqueous solution with a NHS molecule also present in the solution itself. Thus the signal does not come from only solvated C-Dots, but rather from a mixture of solvated NHS molecules and C-Dots. The conclusion of the NHS-C-Dots surface composition remains preliminary, requiring similar study of dry C-Dots.

\section{Balance in energy of the predissociation of $\mathrm{CH}_{3}$ I: a REMPI Probe}

The ideal way to investigate a fast molecular dissociation process is to measure the appearance of the momenta of fragments relative to the center of mass of the parent, for each internal energy of each fragment. Generally, the decisive step is to get an appropriate resonant enhanced multiphoton ionisation (REMPI) pathway. Quite often, the vibrational levels of light molecular fragments are separated enough to enable selective photoionization by a UV probe pulse, even if it is ultrashort and thus possess a broad bandwidth. Figure 19(a) illustrates this for the REMPI-umbrella mode spectra of the $\mathrm{CH}_{3}$ radical through the $3 \mathrm{p}_{z}^{2} A{ }_{2}$ state. A selective photoionization of the fragment as a function of its internal energy based on these resonances can then be done in the femtosecond regime. It is also worth noting that a $200 \mathrm{~cm}^{-1}(25 \mathrm{meV})$ probe bandwidth is sufficiently broad to overlap the entire $\mathrm{Q}$ rotational branch within each umbrella band, and that the 75 fs probe pulse duration is short enough to compete efficiently with the predissociation of the intermediate $3 \mathrm{p}_{z}^{2} A{ }_{2}$ state Rydberg state into $\mathrm{CH}_{2}+\mathrm{H}$. The fs-REMPI of the fragments provides valuable insight into the predissociation. As a proof-of-concept case study, we have studied the predissociation of the vibrationless level of the B-6s[2] state by using time-resolved photoion velocity map imaging ${ }^{86}$. The energy diagram of this predissociation as well as the different UV probe wavelengths required to state-selectively ionized $\mathrm{CH}_{3}$ produced in several quanta in the umbrella mode are shown in figure 19(b).

The UV tunability required for the probe pulse is obtained by frequency mixing the NOPA output (compressed pulses with $25 \mu \mathrm{J}$ of energy tunable between 510 and $700 \mathrm{~nm}$ ), with the $800 \mathrm{~nm}$ fundamental beam to produce $7 \mu \mathrm{J} /$ pulse in the UV range. This UV resonant probe is focused on the molecular beam with a $200 \mathrm{~mm}$ fused silica lens, leading to an intensity of $<5 \times 10^{-11}$ $\mathrm{W} / \mathrm{cm}^{2}$. The vibrational branching fractions of the $\mathrm{CH}_{3}$ are recovered for each pump-probe delay by fitting the kinetic energy distribution recorded by using nonresonant (403 $\mathrm{nm}$ probe wavelength) multiphoton ionization of either the I or the $\mathrm{CH}_{3}$ photofragment with the individual vibrationally state-selected $\mathrm{CH}_{3}$ distributions. Fig $19(\mathrm{~d})$ illustrates the quality of the fit for a 8 ps pump-probe delay when the predissociation dynamics is terminated. The fs-REMPI allows a direct visualization of the complete evolution at the femtosecond scale of photodissociation in small molecular systems.

\section{CONCLUSION}

In conclusion, the Aurore facility offers a wide range of scientific opportunities ranging from picosecond to femtosecond solid state dynamics, down to attosecond timescale in dilute matter. As most of the experiments performed are extremely demanding, the stability and reliability of the laser source is of crucial importance. This is achieved primarily by the careful characterisation of the laser parameters online and a continuous improvement process taking advantage of the last technological developments and the users feedback. In this sense the platform is working as other light sources like synchrotrons, where machines, beamlines and experiments are designed and operated by dedicated specialized persons. As future improvement, the next step will be the implementation of an adaptive mirror, coupled with a Shack-Hartmann sensor, to enhance the wavefront quality of the Aurore laser beam.

\section{DATA AVAILABILITY STATEMENT}

The data that supports the findings of this study are either available within the article or available from the corresponding author upon reasonable request.

\section{ACKNOWLEDGMENTS}

All the authors are grateful to the ANR (CASTORS project ANR-13-JS04-0002, HarMoDyn project ANR08-JCJC-0029, MISFITS project ANR-14-CE32-0014, through the Program IdEx Bordeaux - LAPHIA (ANR10-IDEX-03-02) and the Cluster of Excellence in Plasmas Physics in Paris, PLAS@PAR, (ANR-11-IDEX-0004-02) and the Region Nouvelle Aquitaine (20091304003 ATTOMOL and 2.1.3-09010502 COLA2 projects) for financial supports. Experiment on C-Dots has also received funding from the European Union's Horizon 2020 research and innovation programme under grant agreement no 654148 Laserlab-Europe. S.B. acknowledges the Natural Sciences and Engineering Research Council of Canada Vanier Scholarship. M. de A.V. thanks the Mexican Council for Science and Technology (Grant 611509).

${ }^{1}$ G. F. Albrecht, J. M. Eggleston, and J. J. Ewing, Optics Communications 52, 401 (1985).

${ }^{2}$ P. Lacovara, L. Esterowitz, and R. Allen, Optics Letters 10, 273 (1985).

${ }^{3}$ P. F. Moulton, JOSA B 3, 125 (1986)

${ }^{4}$ D. Strickland and G. Mourou, Optics Communications 55, 447 (1985). 

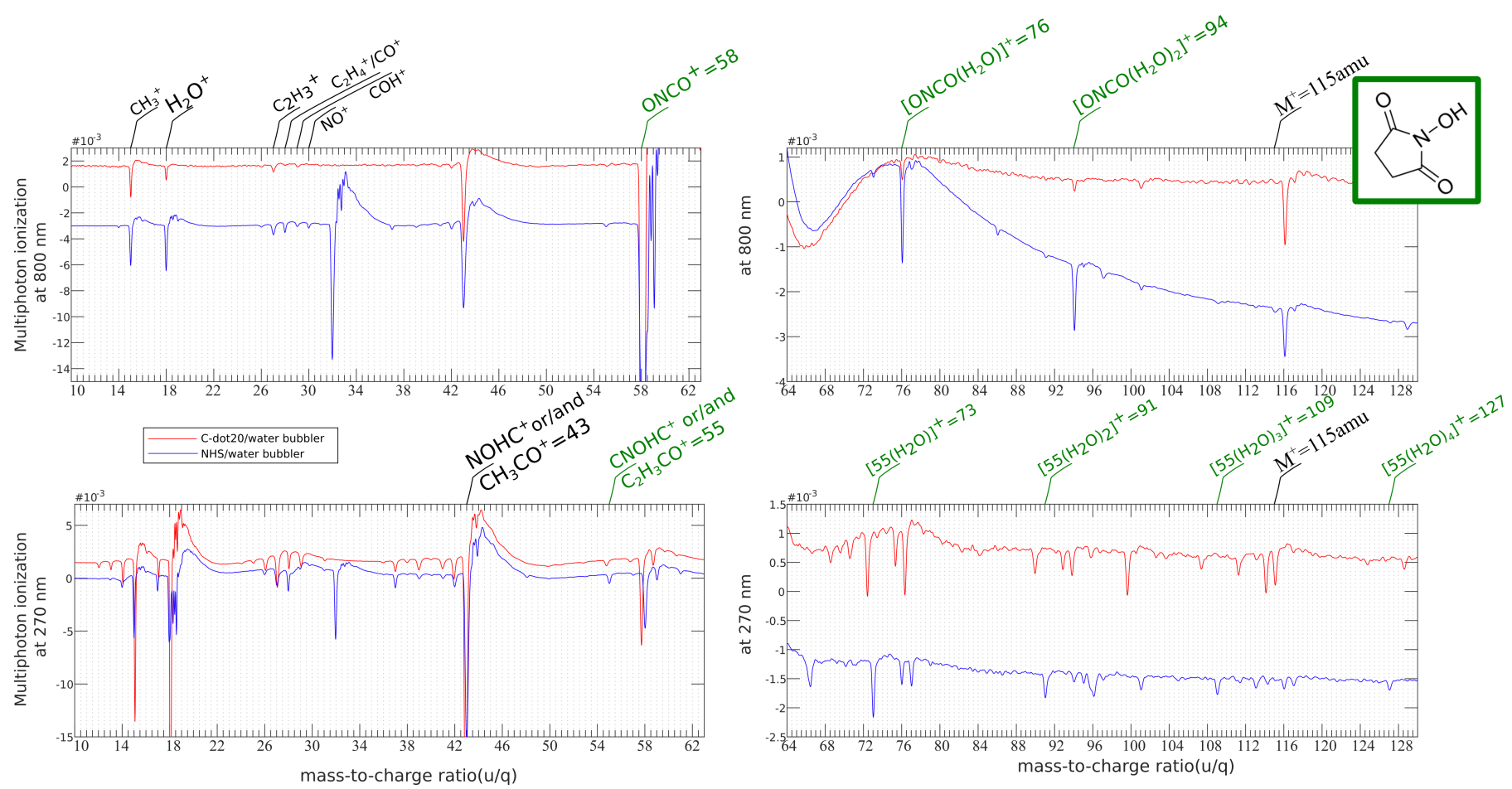

FIG. 18. Mass spectrum measured on C-Dots and NHS initiated by multiphoton ionization at $270 \mathrm{~nm}$ excitation wavelength (lower graphs) and at $800 \mathrm{~nm}$ excitation wavelength (upper graphs)

${ }^{5}$ G. Steinmeyer, D. H. Sutter, L. Gallmann, N. Matuschek, and U. Keller, Science 286, 1507 (1999).

${ }^{6}$ N. Fedorov, G. Geoffroy, G. Duchateau, L. Stolcova, J. Proska, F. Novotny, M. Domonkos, H. Jouin, P. Martin, and M. Raynaud, J. Phys.: Condens. Matter 28, 315301 (2016).

${ }^{7}$ C. Fourment, F. Deneuville, D. Descamps, F. Dorchies, S. Petit, O. Peyrusse, B. Holst, and V. Recoules, Phys. Rev. B 89, 161110 (2014).

${ }^{8}$ A. Comby, S. Beaulieu, M. Boggio-Pasqua, D. Descamps, F. Légaré, L. Nahon, S. Petit, B. Pons, B. Fabre, Y. Mairesse, and V. Blanchet, The Journal of Physical Chemistry Letters 7, 4514 (2016), pMID: 27786493 , https://doi.org/10.1021/acs.jpclett.6b02065.

${ }^{9}$ S. Beaulieu, A. Comby, A. Clergerie, J. Caillat, D. Descamps, N. Dudovich, B. Fabre, R. Géneaux, F. Légaré, S. Petit, B. Pons, G. Porat, T. Ruchon, R. Taïeb, V. Blanchet, and Y. Mairesse, Science 358, 1288 (2017), http://science.sciencemag.org/content/358/6368/1288.full.pdf.

${ }^{10}$ G. Chériaux, P. Rousseau, F. Salin, J. P. Chambaret, B. Walker, and L. F. Dimauro, Opt. Lett. 21, 414 (1996).

${ }^{11}$ C. G. Durfee, S. Backus, M. M. Murnane, and H. C. Kapteyn, IEEE Journal of Selected Topics in Quantum Electronics 4, 395 (1998).

12 E. B. Treacy, IEEE J. Quantum Electronics 5, 454 (1969).

${ }^{13} \mathrm{~S}$. Kane and J. Squier, J. Opt. Soc. Am. B 14, 1237 (1997)

${ }^{14}$ C. Hooker, Y. Tang, O. Chekhlov, J. Collier, E. Divall, K. Ertel,

S. Hawkes, B. Parry, and P. P. Rajeev, Opt. Express 19, 2193 (2011).

${ }^{15}$ Z. Li, S. Tokita, S. Matsuo, K. Sueda, T. Kurita, T. Kawasima, and N. Miyanaga, Opt. Express 25, 21201 (2017).

${ }^{16}$ A. McPherson, G. Gibson, H. Jara, U. Johann, T. S. Luk, I. McIntyre, K. Boyer, and C. K. Rhodes, J. Opt. Soc. Am B 4, 595 (1987).

${ }^{17}$ M. Ferray, A. L'Huillier, X. F. Li, L. A. Lompre, G. Mainfray, and C. Manus, Journal of Physics B: Atomic, Molecular and Optical Physics 21, L31 (1988)

${ }^{18}$ L. Le Déroff, P. Salières, B. Carré, D. Joyeux, and D. Phalippou, Physical Review A 61, 043802 (2000).

${ }^{19}$ J.-F. Hergott, M. Kovacev, H. Merdji, C. Hubert, Y. Mairesse, E. Jean, P. Breger, P. Agostini, B. Carré, and P. Salières, Phys- ical Review A 66, 021801 (2002).

${ }^{20}$ E. Takahashi, Y. Nabekawa, and K. Midorikawa, Opt. Lett. 27, 1920 (2002).

${ }^{21}$ S. Hädrich, A. Klenke, J. Rothhardt, M. Krebs, A. Hoffmann, O. Pronin, V. Pervak, J. Limpert, and A. Tünnermann, Nature Photonics 8, 779 (2014).

${ }^{22}$ A. Comby, D. Descamps, S. Beauvarlet, A. Gonzalez, F. Guichard, S. Petit, Y. Zaouter, and Y. Mairesse, Opt. Express 27, 20383 (2019).

${ }^{23}$ Y. Mairesse, O. Gobert, P. Breger, H. Merdji, P. Meynadier, P. Monchicourt, M. Perdrix, P. Salières, and B. Carré, Physical Review Letters 94 (2005), 10.1103/PhysRevLett.94.173903.

${ }^{24}$ T. Sekikawa, T. Kanai, and S. Watanabe, Phys. Rev. Lett. 91, 103902 (2003).

${ }^{25}$ P. M. Paul, E. S. Toma, P. Breger, G. Mullot, P. Balcou, H. G. Muller, and P. Agostini, Science 292, 1689 (2001).

${ }^{26}$ M. Hentschel, R. Kienberger, C. Spielmann, G. A. Reider, N. Milosevic, T. Brabec, P. Corkum, U. Heinzmann, M. Drescher and F. Krausz, Nature 414, 509 (2001).

${ }^{27}$ F. Krausz and M. Ivanov, Reviews of Modern Physics 81, 163 (2009).

${ }^{28}$ J. Bourgalais, N. Carrasco, L. Vettier, T. Gautier, V. Blanchet, S. Petit, D. Descamps, N. Fedorov, R. Delos, and J. Gaudin, 10, 10009

${ }^{29}$ M. Lein, N. Hay, R. Velotta, J. Marangos, and P. Knight, Physical Review A 66, 023805 (2002).

${ }^{30}$ J. Itatani, J. Levesque, D. Zeidler, H. Niikura, H. Pepin, J. Kieffer, P. Corkum, and D. Villeneuve, Nature 432, 867 (2004).

${ }^{31} \mathrm{~S}$. Bohman, A. Suda, T. Kanai, S. Yamaguchi, and K. Midorikawa, Opt. Lett. 35, 1887 (2010).

${ }^{32}$ V. Cardin, N. Thir, S. Beaulieu, V. Wanie, F. Lgar, and B. E. Schmidt, Applied Physics Letters 107, 181101 (2015), https://doi.org/10.1063/1.4934861.

${ }^{33}$ A. Ferré, A. E. Boguslavskiy, M. Dagan, V. Blanchet, B. D. Bruner, F. Burgy, A. Camper, D. Descamps, B. Fabre, N. Fedorov, J. Gaudin, G. Geoffroy, J. Mikosch, S. Patchkovskii, S. Petit, T. Ruchon, H. Soifer, D. Staedter, I. Wilkinson, A. Stolow, N. Dudovich, and Y. Mairesse, Nat. Commun. 6 (2015), 10.1038/ncomms6952.

${ }^{34}$ A. Ferré, C. Handschin, M. Dumergue, F. Burgy, A. Comby, 


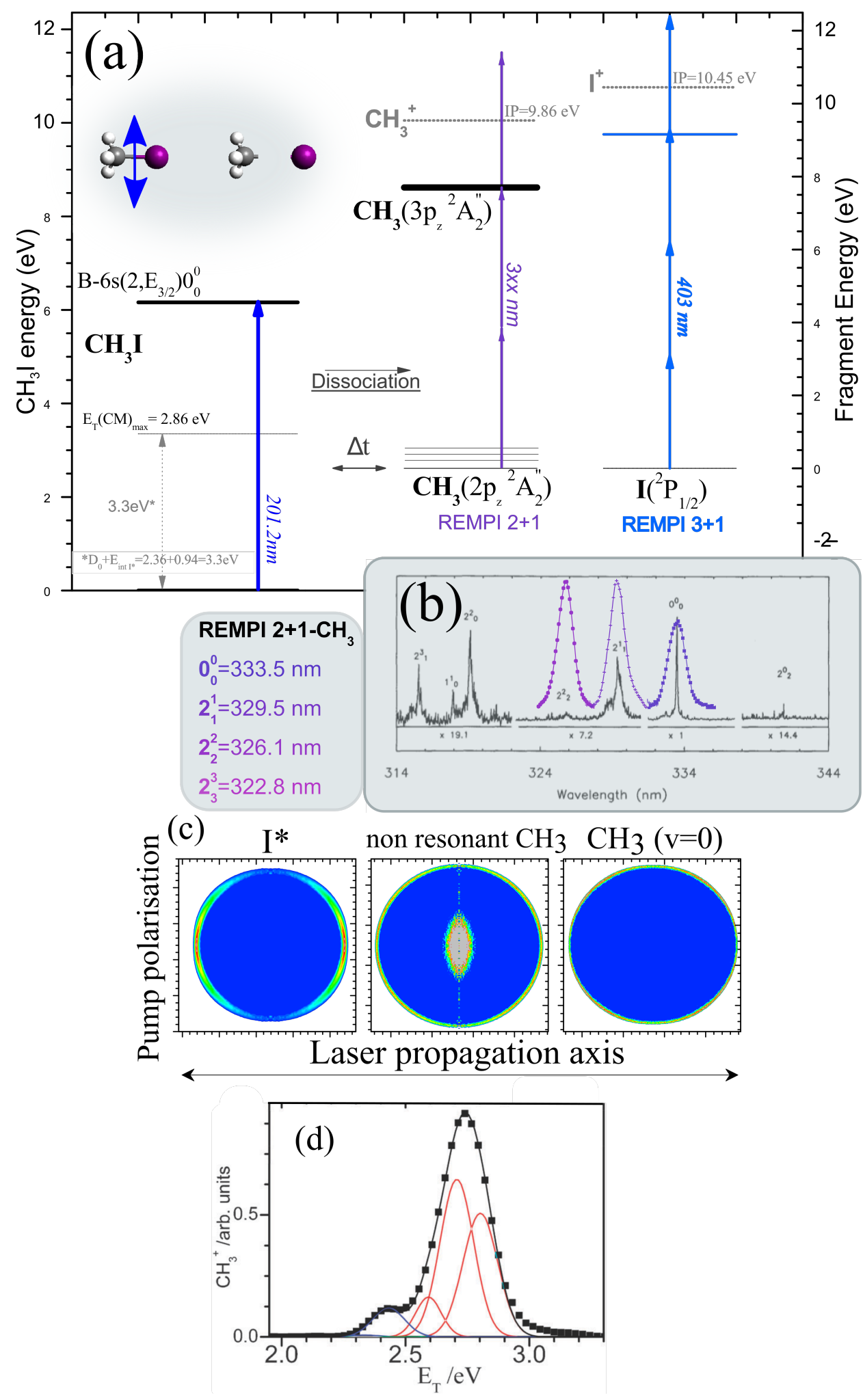

FIG. 19. $\mathrm{CH}_{3} \mathrm{I}$ predissociation from the origin band of the first Rydberg state. (a) Energy diagram of the $\mathrm{CH}_{3} \mathrm{I}$ predissociation. (b) The wavelength tunability of the NOPA allows to selectively photoionize the fragments $\mathrm{CH}_{3}$ as a function of its umbrella mode activity by a $2+1 \mathrm{REMPI}^{93}$. The umbrella quantum $(\sim 75 \mathrm{meV})$ is large enough to support a selective ionisation along this vibrational mode, while the $25 \mathrm{meV}$ bandwidth of the probe pulse is large enough to ionize all the rotational levels populated by the predissociation.(c) Image of iodine, $\mathrm{CH}_{3}$ recorded off resonance, independently of their vibration and rotation and $\mathrm{CH}_{3}$ $(\mathrm{v}=0)$ produced by the predissociation and resonantly ionised at $333.5 \mathrm{~nm}$ through the $3 \mathrm{p}_{z}^{2} A{ }_{2}{ }_{2}$ state. Since the dipole moment of this electronic transition is perpendicular to the C-I bond and the predissociation in the picosecond range, the fragments are mainly produced perpendicular to the pump polarisation. (d) $\mathrm{CH}_{3}$ kinetic energy distribution recorded off resonance (scatter plot recorded $403 \mathrm{~nm}$ probe wavelength) and $\mathrm{CH}_{3}$ kinetic energy distribution recorded through $2+1$ resonance by tuning the probe wavelength along the REMPI spectra shown in (a), the internal energy distribution of $\mathrm{CH}_{3}$ fragments can be retrieved along the predissociation dynamics. Here are shown the distributions at a pump-probe delay of 8 ps, once all the dynamics is finished. 
D. Descamps, B. Fabre, G. A. Garcia, R. Géneaux, L. Merceron, E. Mével, L. Nahon, S. Petit, B. Pons, D. Staedter, S. Weber T. Ruchon, V. Blanchet, and Y. Mairesse, Nat. Photon. 9, 93 (2015).

35 A. Camper, A. Ferré, V. Blanchet, F. Burgy, D. Descamps, S. Petit, T. Ruchon, and Y. Mairesse, Opt. Lett. 40, 5387 (2015).

${ }^{36}$ H. Ruf, C. Handschin, R. Cireasa, N. Thiré, A. Ferré, S. Petit D. Descamps, E. Mével, E. Constant, V. Blanchet, B. Fabre, and Y. Mairesse, Physical Review Letters 110, 083902 (2013).

${ }^{37}$ R. Cireasa, A. E. Boguslavskiy, B. Pons, M. C. H. Wong, D. Descamps, S. Petit, H. Ruf, N. Thiré, A. Ferré, J. Suarez J. Higuet, B. E. Schmidt, A. F. Alharbi, F. Légaré, V. Blanchet, B. Fabre, S. Patchkovskii, O. Smirnova, Y. Mairesse, and V. R. Bhardwaj, Nature Physics 11, 654 (2015).

${ }^{38}$ H. Ruf, C. Handschin, A. Ferré, N. Thiré, J. B. Bertrand, L. Bonnet, R. Cireasa, E. Constant, P. B. Corkum, D. Descamps, B. Fabre, P. Larregaray, E. Mével, S. Petit, B. Pons, D. Staedter, H. J. Worner, D. M. Villeneuve, Y. Mairesse, P. Halvick, and V. Blanchet, Journal of Chemical Physics 137 (2012).

${ }^{39}$ S. Beaulieu, E. Bloch, L. Barreau, A. Comby, D. Descamps, R. Géneaux, F. Légaré, S. Petit, and Y. Mairesse, Phys. Rev. A 95, 041401 (2017).

${ }^{40}$ P. Çarçabal, D. Descamps, S. Petit, Y. Mairesse, V. Blanchet, and R. Cireasa, Faraday Discuss. (2016), 10.1039/C6FD00129G.

${ }^{41}$ F. Catoire, A. Ferré, O. Hort, A. Dubrouil, L. Quintard,

D. Descamps, S. Petit, F. Burgy, E. Mével, Y. Mairesse, and E. Constant, Physical Review A 94 (2016).

${ }^{42}$ S. Beaulieu, A. Ferré, R. Geneaux, R. Canonge, D. Descamps, B. Fabre, N. Fedorov, F. Légaré, S. Petit, T. Ruchon, V. Blanchet, Y. Mairesse, and B. Pons, New Journal of Physics 18 (2016).

${ }^{43}$ S. Beaulieu, A. Comby, A. Clergerie, J. Caillat, D. Descamps, N. Dudovich, B. Fabre, R. Géneaux, F. Légaré, S. Petit, B. Pons G. Porat, T. Ruchon, R. Taïeb, V. Blanchet, and Y. Mairesse, Science 358, 1288 (2017).

${ }^{44}$ F. Ferrari, F. Calegari, M. Lucchini, C. Vozzi, S. Stagira, G. Sansone, and M. Nisoli, Nature Photonics 4, 875 (2010).

${ }^{45}$ E. Goulielmakis, M. Schultze, M. Hofstetter, V. Yakovlev, J. Gagnon, M. Uiberacker, A. Aquila, E. Gullikson, D. Attwood, R. Kienberger, F. Krausz, and U. Kleineberg, Science 320, 1614 (2008).

${ }^{46}$ G. Sansone, E. Benedetti, F. Calegari, C. Vozzi, L. Avaldi, R. Flammini, L. Poletto, P. Villoresi, C. Altucci, R. Velotta, S. Stagira, S. De Silvestri, and M. Nisoli, Science 314, 443 (2006).

${ }^{47}$ H. Mashiko, S. Gilbertson, C. Li, S. Khan, M. Shakya, E. Moon, and Z. Chang, Physical Review Letters 100, 103906 (2008).

${ }^{48}$ H. Vincenti and F. Quéré, Phys. Rev. Lett. 108, 113904 (2012).

${ }^{49}$ T. J. Hammond, G. G. Brown, K. T. Kim, D. M. Villeneuve, and P. B. Corkum, Nature Photonics 10, 171 (2016).

${ }^{50}$ S. Beaulieu, S. Camp, D. Descamps, A. Comby, V. Wanie, S. Petit, F. Légaré, K. J. Schafer, M. B. Gaarde, F. Catoire, and Y. Mairesse, Physical Review Letters 117 (2016).

${ }^{51}$ K. T. Kim, C. Zhang, T. Ruchon, J.-F. Hergott, T. Auguste, D. M. Villeneuve, P. B. Corkum, and F. Quéré, Nature Photonics 7, 651 (2013)

${ }^{52}$ K. Bennemann, Annalen der Physik 18, 480 (2009).

${ }^{53}$ C. Fourment, B. Chimier, F. Deneuville, D. Descamps, F. Dorchies, G. Duchateau, M.-C. Nadeau, and S. Petit, Phys. Rev. B 98, 155110 (2018).

${ }^{54}$ J. P. Colombier, P. Combis, F. Bonneau, R. Le Harzic, and E. Audouard, Phys. Rev. B 71, 165406 (2005).

${ }^{55}$ C. Froehly, A. Lacourt, and J. C. Vienot, OJ. Opt. (Paris) 4 183 (1973).

${ }^{56}$ J. P. Geindre, P. Audebert, A. Rousse, F. Fallies, J. C. Gauthier, A. Mysyrowicz, A. D. Santos, and G. Hamoniaux, Opt. Lett 19, 1997 (1994)

${ }^{57}$ F. Deneuville, B. Chimier, D. Descamps, F. Dorchies, S. Hulin, S. Petit, O. Peyrusse, J. J. Santos, and C. Fourment, Applied Physics Letters 102, 194104 (2013).

${ }^{58}$ P. Noé, C. Vallée, F. Hippert, F. Fillot, and J.-Y. Raty, Semiconductor Science and Technology 33, 013002 (2018).

${ }^{59}$ L. Waldecker, T. A. Miller, M. Rude, R. Bertoni, J. Osmond, V. Pruneri, R. E. Simpson, R. Ernstorfer, and S. Wall, Nature
Materials 14, 991 (2015)

${ }^{60}$ E. Matsubara, S. Okada, T. Ichitsubo, T. Kawaguchi, A. Hirata, P. F. Guan, K. Tokuda, K. Tanimura, T. Matsunaga, M. W. Chen, and N. Yamada, Phys. Rev. Lett. 117, 135501 (2016).

${ }^{61}$ Z. Lin, L. V. Zhigilei, and V. Celli, Phys. Rev. B 77, 075133 (2008).

${ }^{62}$ S. Anisimov and B. Rethfeld,
Nonresonant Laser-Matter Interaction (NLMI-9), in ings of SPIE-the International Society for Optical Engineering, Vol. 3093 (Proceedings of SPIE, 1997) p. 192

${ }^{63}$ Y. V. Petrov, K. P. Migdal, N. A. Inogamov, and V. V. Zhakhovsky, Applied Physics B 119, 401 (2015).

${ }^{64}$ J. Winter, S. Rapp, M. Schmidt, and H. P. Huber, Applied Surface Science 417, 2 (2017), 10th International Conference on Photoexcited Processes and Applications.

${ }^{65}$ M. Kandyla, T. Shih, and E. Mazur, Phys. Rev. B 75, 214107 (2007).

${ }^{66}$ R. Li, K. Sundqvist, J. Chen, H. E. Elsayed-Ali, J. Zhang, and P. M. Rentzepis, Structural Dynamics 5, 044501 (2018) https://doi.org/10.1063/1.5029970.

${ }^{67}$ M. Z. Mo, Z. Chen, R. K. Li, M. Dunning, B. B. L. Witte, J. K. Baldwin, L. B. Fletcher, J. B. Kim, A. Ng, R. Redmer, A. H. Reid, P. Shekhar, X. Z. Shen, M. Shen, K. Sokolowski-Tinten, Y. Y. Tsui, Y. Q. Wang, Q. Zheng, X. J. Wang, and S. H. Glenzer, Science 360, 1451 (2018), http://science.sciencemag.org/content/360/6396/1451.full.pdf.

${ }^{68}$ E. Bévillon, J. P. Colombier, V. Recoules, and R. Stoian, Phys. Rev. B 89, 115117 (2014).

${ }^{69}$ C. A. Mack, Kinetic coefficients for d-band metals in two-temperature sta (Springer, London, 2013).

70 S. Hofmann, Auger- and X-Ray Photoelectron Spectroscopy in Materials (Springer, London, 2013).

${ }^{71}$ M. Aeschlimann, C. A. Schmuttenmaer, H. E. ElsayedAli, R. J. D. Miller, J. Cao, Y. Gao, and D. A. Mantell, The Journal of Chemical Physics 102, 8606 (1995), https://doi.org/10.1063/1.468962.

${ }^{72}$ The photodiode was calibrated at the Physikalisch-Technische Bundesanstalt (PTB) using synchrotron radiation..

${ }^{73}$ S. Hellmann, K. Rossnagel, M. Marczynski-Bühlow, and L. Kipp, Phys. Rev. B 79, 035402 (2009).

${ }^{74}$ A. Fognini, G. Salvatella, T. U. Michlmayr, C. Wetli, U. Ramsperger, T. Bähler, F. Sorgenfrei, M. Beye, A. Eschenlohr, N. Pontius, C. Stamm, F. Hieke, M. Dell'Angela, S. de Jong, R. Kukreja, N. Gerasimova, V. Rybnikov, H. Redlin, J. Raabe, A. Föhlisch, H. A. Drr, W. Wurth, D. Pescia, A. Vaterlaus, and Y. Acremann, New Journal of Physics 16, 043031 (2014).

${ }^{75}$ X. Zhou, B. Wannberg, W. Yang, V. Brouet, Z. Sun, J. Douglas, D. Dessau, Z. Hussain, and Z.-X. Shen, Journal of Electron Spectroscopy and Related Phenomena 142, 27 (2005).

${ }^{76}$ M. Dell'Angela, F. Hieke, F. Sorgenfrei, N. Gerken, M. Beye, N. Gerasimova, H. Redlin, and W. Wurth, Surface Science 643, 197 (2016), present challenges in surface science, a special issue in honour of Dietrich Menzel.

${ }^{77}$ R. Al-Obaidi, M. Wilke, M. Borgwardt, J. Metje, A. Moguilevski, N. Engel, D. Tolksdorf, A. Raheem, T. Kampen, S. Mahl, I. Y. Kiyan, and E. F. Aziz, New Journal of Physics 17, 093016 (2015).

${ }^{78}$ L.-P. Oloff, M. Oura, K. Rossnagel, A. Chainani, M. Matsunami, R. Eguchi, T. Kiss, Y. Nakatani, T. Yamaguchi, J. Miyawaki, M. Taguchi, K. Yamagami, T. Togashi, T. Katayama, K. Ogawa, M. Yabashi, and T. Ishikawa, New Journal of Physics 16, 123045 (2014).

${ }^{79}$ A. Belsky, N. Fedorov, S. Gridin, A. Gektin, P. Martin D. Spassky, and A. Vasil'ev, Radiation Measurements 124, 1 (2019).

${ }^{80}$ S. Beaulieu, A. Comby, D. Descamps, B. Fabre, G. Garcia, R. Géneaux, A. Harvey, F. Légaré, Z. Man, L. Nahon, A. Ordonez, S. Petit, B. Pons, O. Mairesse, Y. Smirnova, and V. Blanchet, Nature Physics 14, 48489 (2018).

${ }^{81}$ N. Thiré, R. Cireasa, V. Blanchet, and S. Pratt, Physical Chemistry Chemical Physics 12, 15644 (2010).

${ }^{82}$ P. Piecuch, J. Hansen, D. Staedter, S. Faure, and V. Blanchet, The Journal of Chemical Physics 138, 201102 (2013).

${ }^{83}$ D. Staedter, N. Thiré, L. Polizzi, Y. Mairesse, P. Mayer, and V. Blanchet, The Journal of Chemical Physics 142, 194306 
(2015).

${ }^{84}$ J. A. Noble, C. Aupetit, D. Descamps, S. Petit, A. Simon, J. Mascetti, N. Ben Amor, and V. Blanchet, Physical Chemistry Chemical Physics (2019), 10.1039/C8CP06895J.

${ }^{85}$ R. Cireasa, J.-B. Hamard, C. Maury, and V. Blanchet, Physica Scripta 80, 048106 (2009).

${ }^{86}$ N. Thiré, D. Staedter, R. Cireasa, V. Blanchet, and S. Pratt, Physical Chemistry Chemical Physics 13, 18485 (2011).

${ }^{87}$ D. Staedter, N. Thiré, E. Baynard, P. C. Samartzis, and V. Blanchet, Phys. Chem. Chem. Phys. 16, 540 (2014).

${ }^{88}$ K. Luria, W. Christen, and U. Even, The Journal of Physical Chemistry A 115, 7362 (2011).

${ }^{89}$ I. Papagiannouli, M. Patanen, V. Blanchet, J. D. Bozek, M. de Anda Villa, M. Huttula, E. Kokkonen, E. Lamour, E. Mével, E. Pelimanni, A. Scalabre, M. Trassinelli, D. M. Bas- sani, A. Lévy, and J. Gaudin, The Journal of Physical Chemistry C 122, 14889 (2018), https://doi.org/10.1021/acs.jpcc.8b03800. ${ }^{90}$ M. De Anda Villa, J. Gaudin, D. Amans, F. Boudjada, J. Bozek, R. Evaristo Grisenti, E. Lamour, G. Laurens, S. Mac, C. Nicolas, I. Papagiannouli, M. Patanen, C. Prigent, E. Robert, S. Steydli, M. Trassinelli, D. Vernhet, and A. Lvy, Langmuir 35, 11859 (2019).

${ }^{91}$ C. S. Stan, C. Albu, A. Coroaba, M. Popa, and D. Sutiman, J Mater. Chem. C 3, 789 (2015)

${ }^{92}$ R. Mota, R. Parafita, A. Giuliani, M.-J. Hubin-Franskin, J. Loureno, G. Garcia, S. Hoffmann, N. Mason, P. Ribeiro, M. Raposo, and P. Limo-Vieira, Chemical Physics Letters 416 152 (2005).

${ }^{93}$ R. Loo, H. Haerri, G. Hall, and P. Houston, The Journal of Chemical Physics 90, 4222 (1989). 


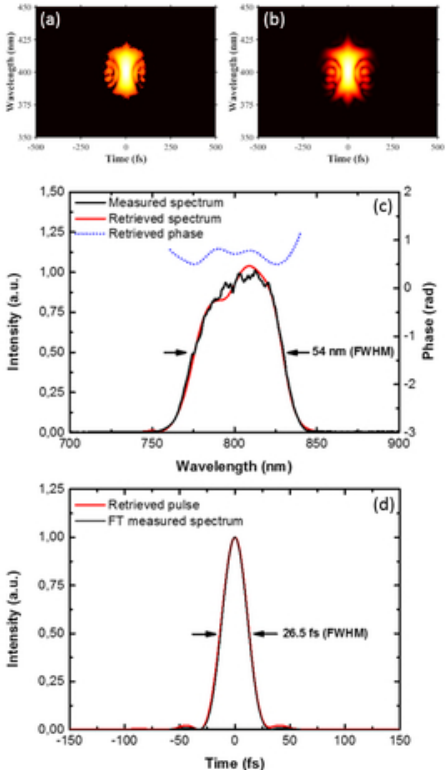


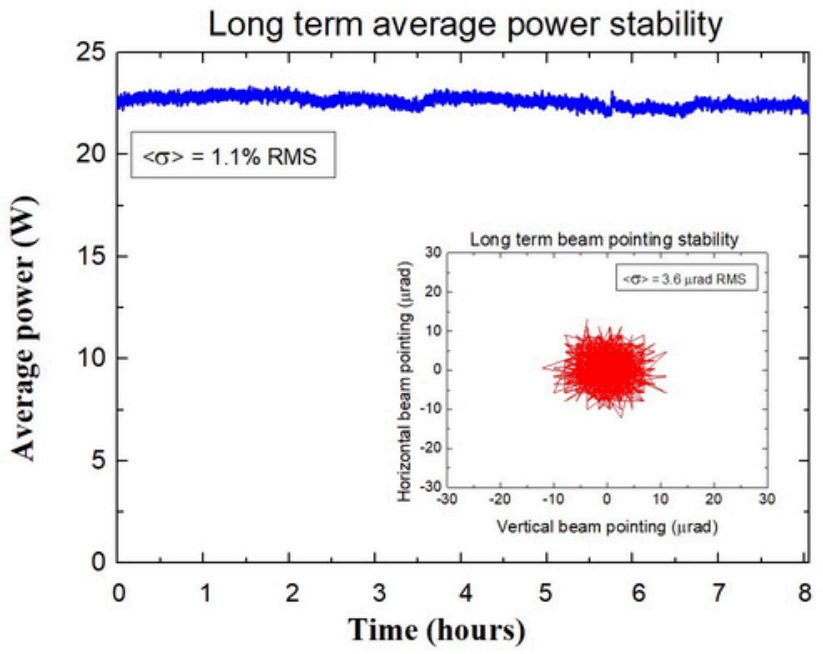




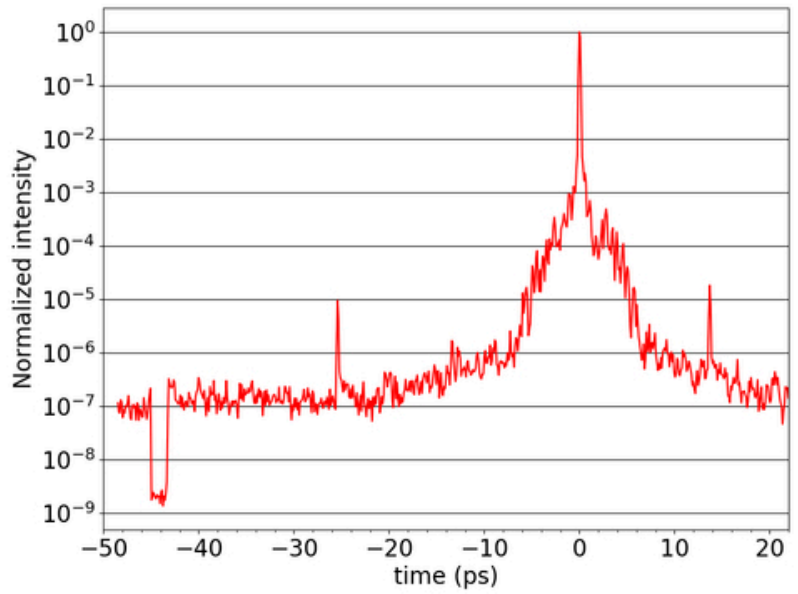




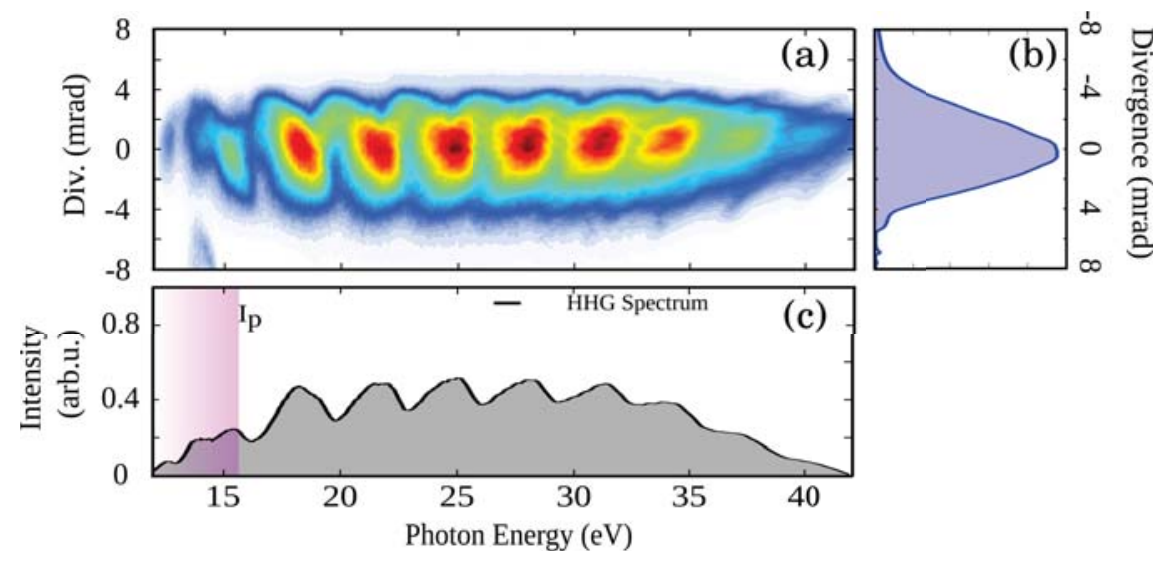




\section{Shot 1}

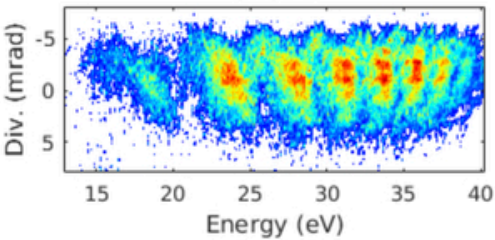

Shot 4

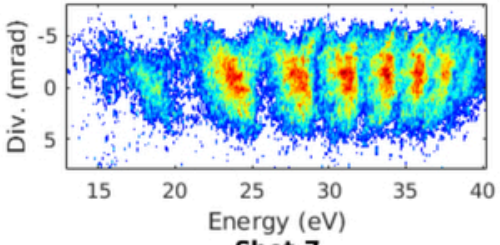

Shot 7

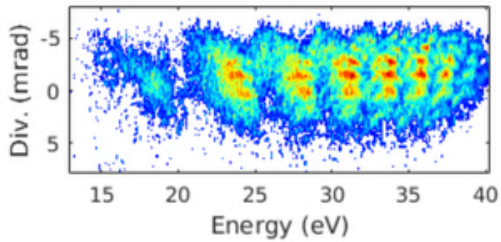

Shot 2

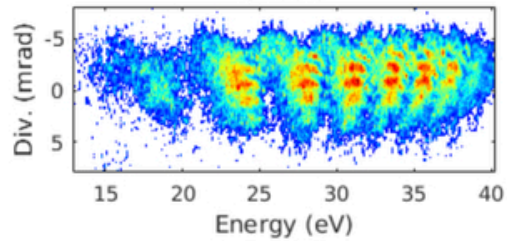

Shot $\mathbf{5}$

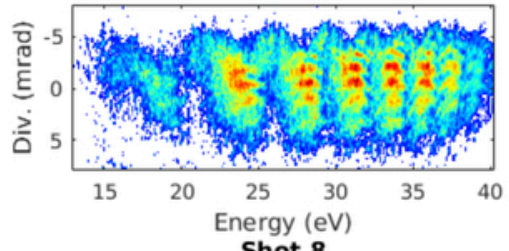

Shot 8

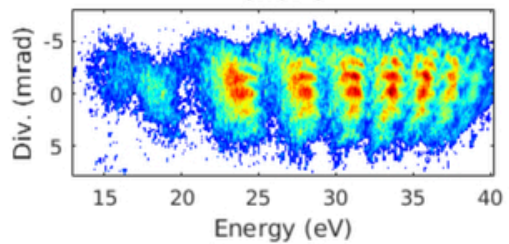

Shot 3

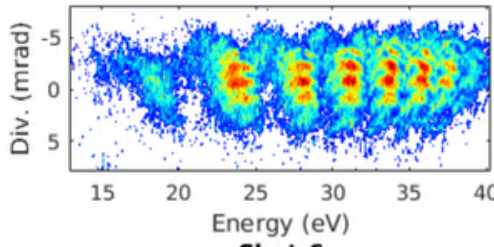

Shot 6

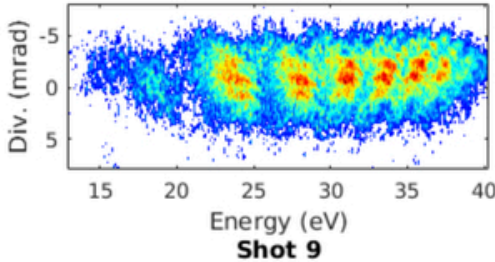

Shot 9

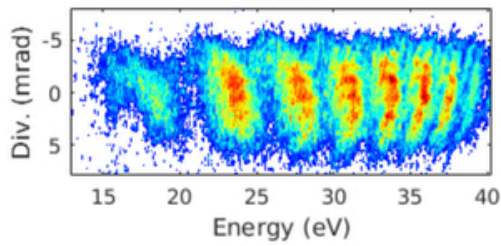




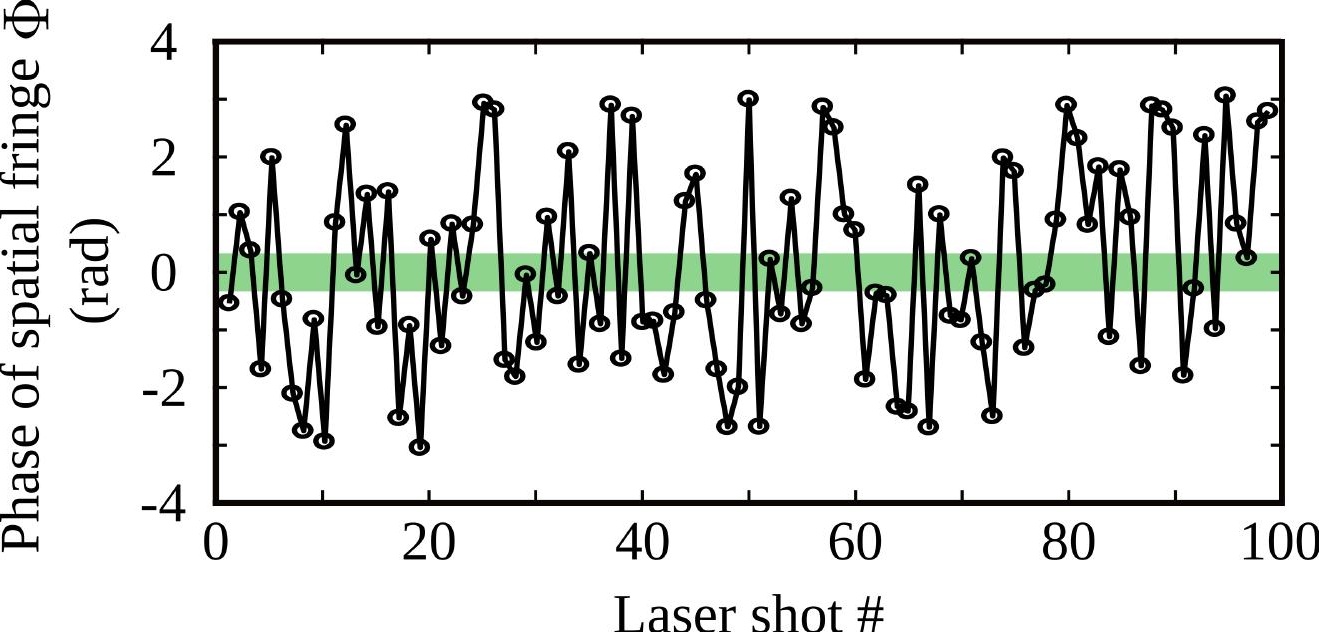




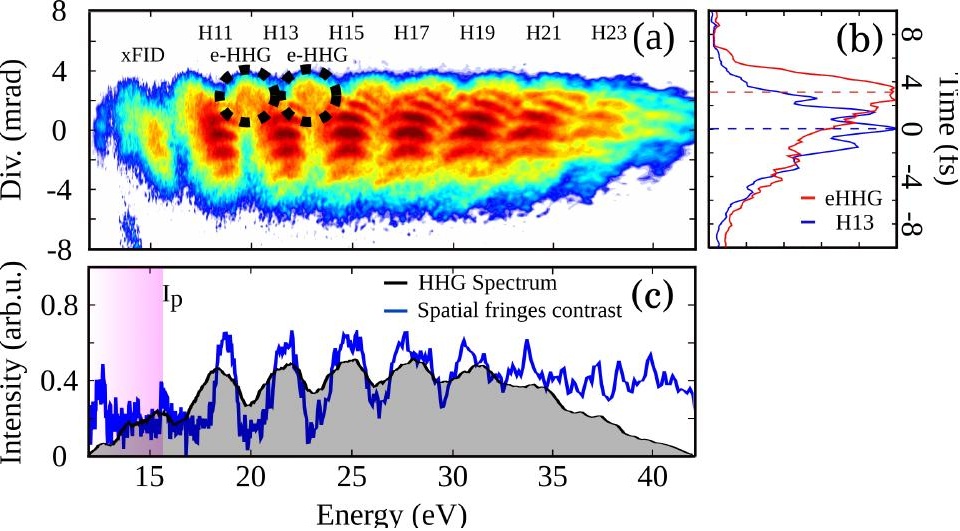


$532 \mathrm{~nm}$ $10 \mu \mathrm{J}$

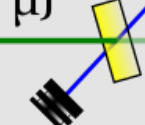

\section{$\mathrm{BBO} 3$}

L
BS
$400 \mathrm{~nm}$

BBO1

\section{BS}

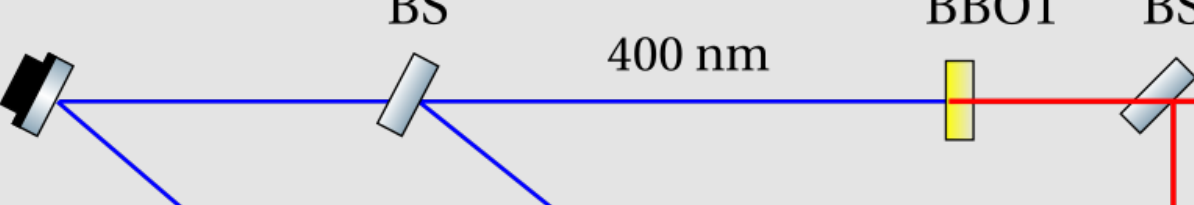

SM

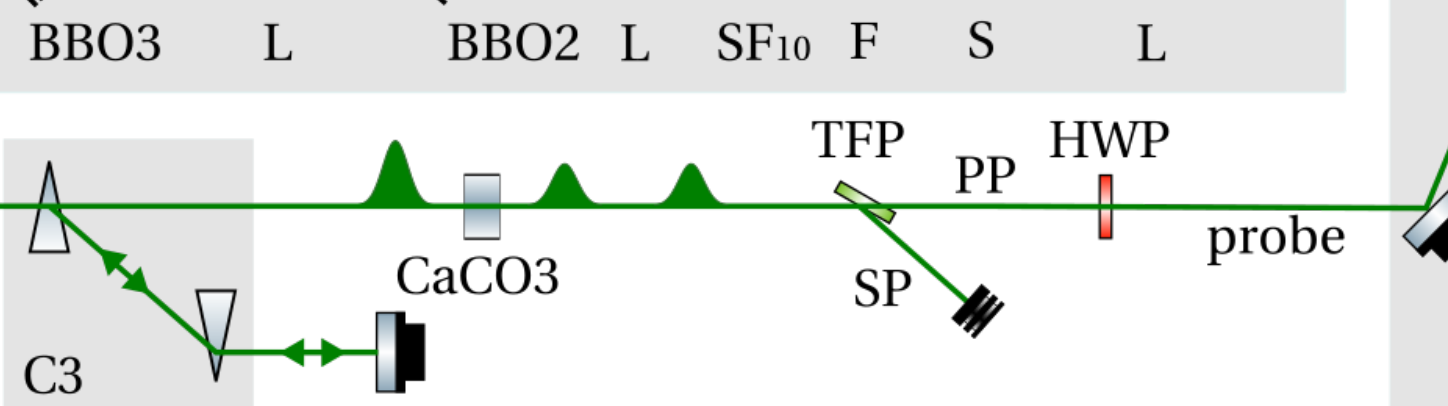

$532 \mathrm{~nm}$ $1 \mu \mathrm{J}$

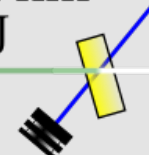

0
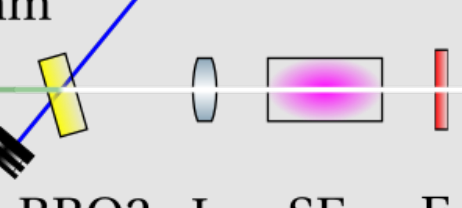

$\mathrm{BBO} 2$

C3

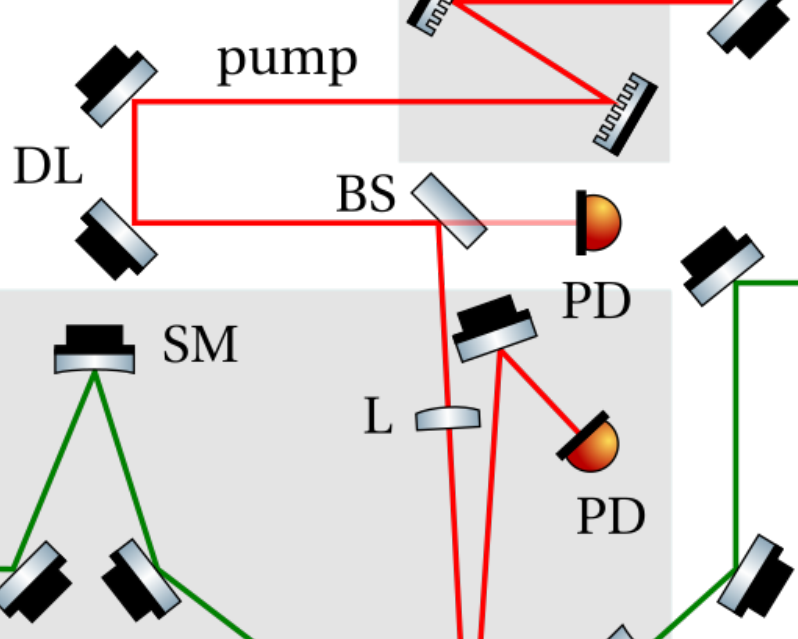

INTERACTION

sample

ADL CHAMBER 
$(55 \pm 3) \mathrm{mJ} / \mathrm{cm}^{2}$ $(101 \pm 4) \mathrm{mJ} / \mathrm{cm}^{2}$
$(150 \pm 4) \mathrm{mJ} / \mathrm{cm}^{2}$

$(202 \pm 2) \mathrm{mJ} / \mathrm{cm}^{2}$
$(251 \pm 3) \mathrm{mJ} / \mathrm{cm}^{2}$

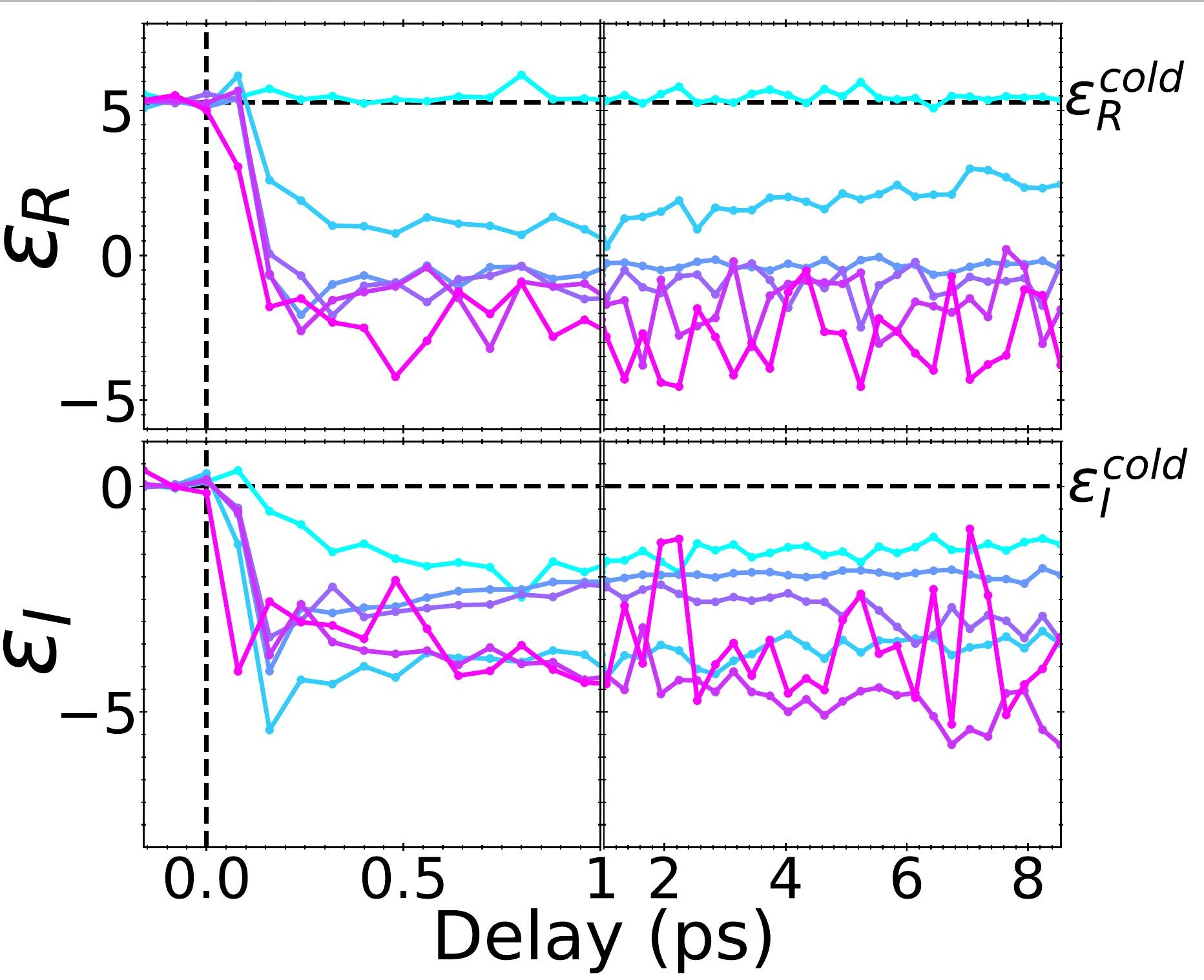




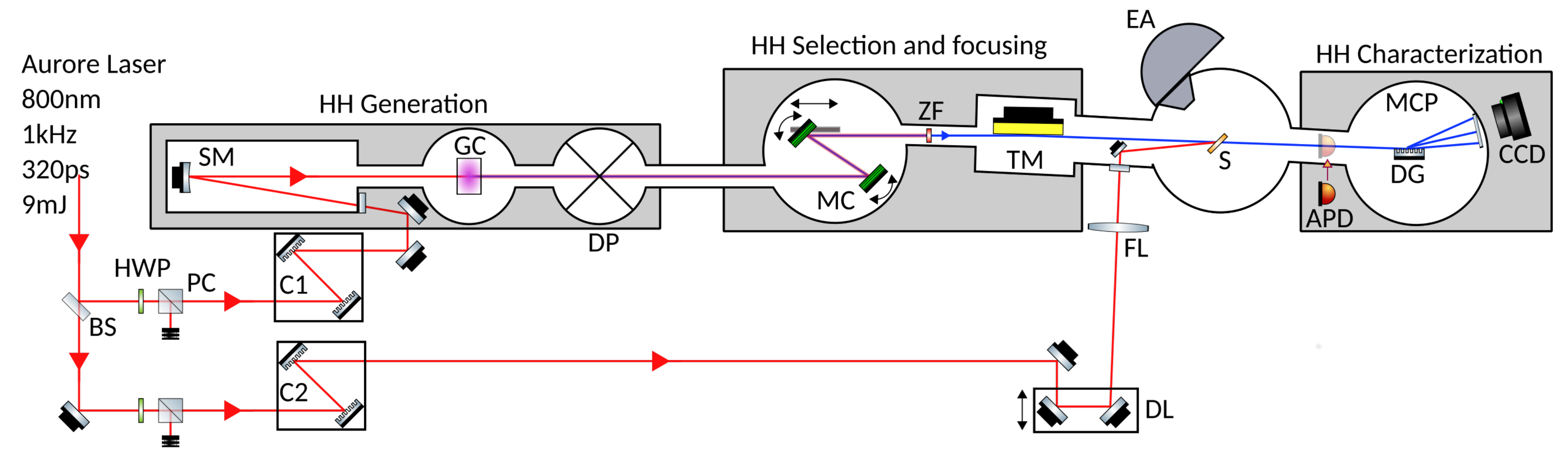




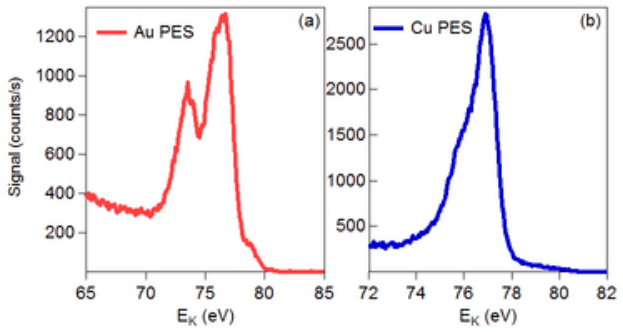




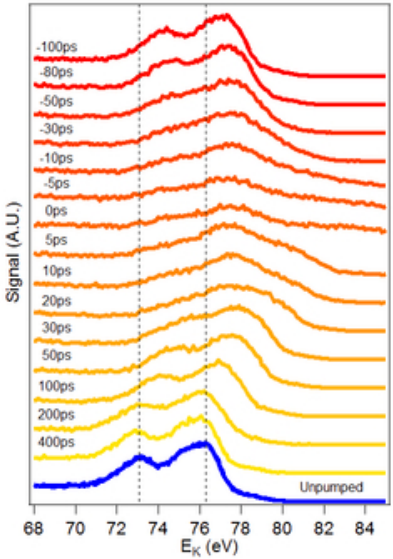


$25 \mathrm{fs}, 9 \mathrm{~mJ}$

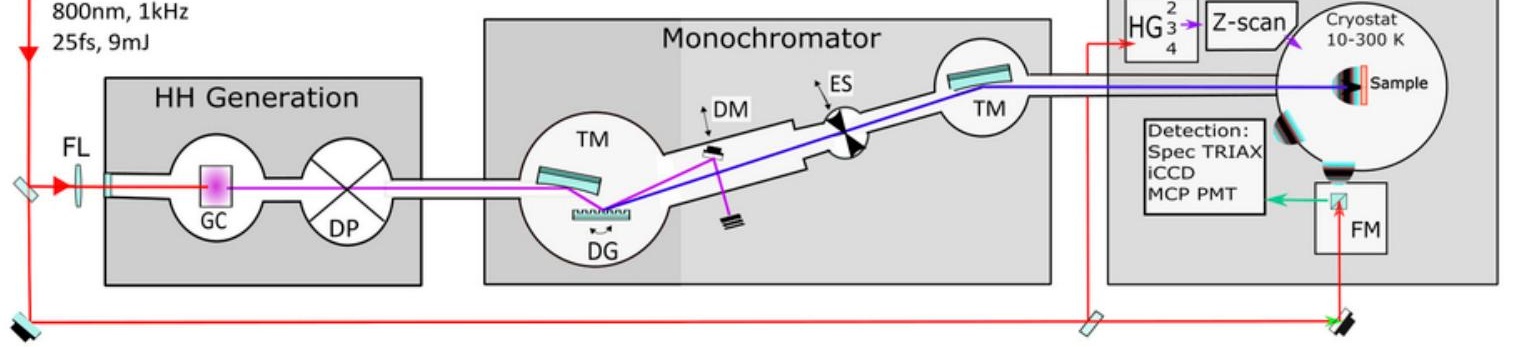




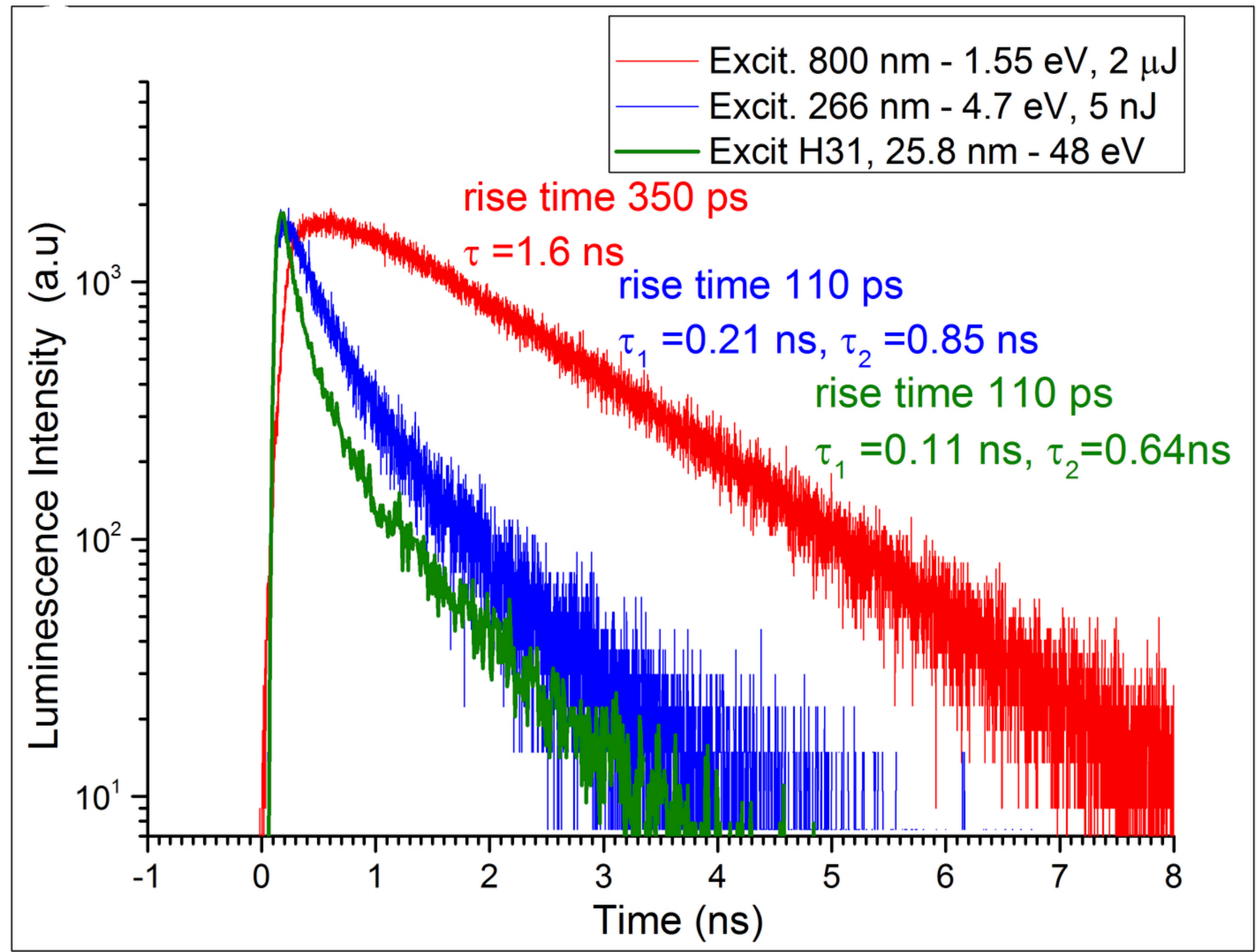




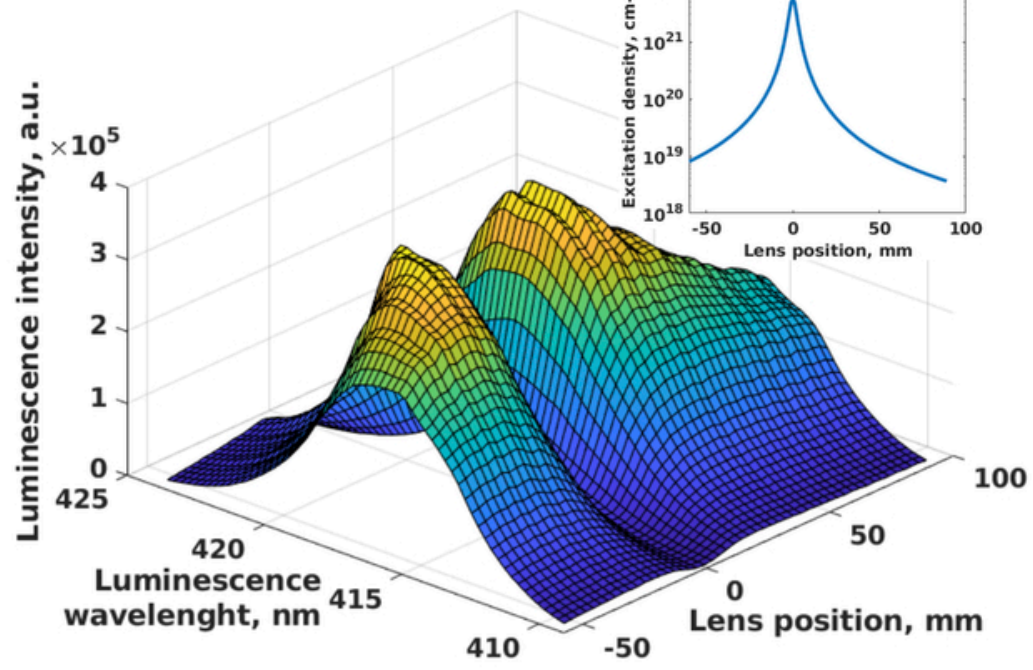




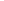




\section{$\approx(\mathrm{c})$ \\ non resonant $\mathrm{CH}_{3} \quad \mathrm{CH}_{3}(\mathrm{v}=0)$ \\ I*}
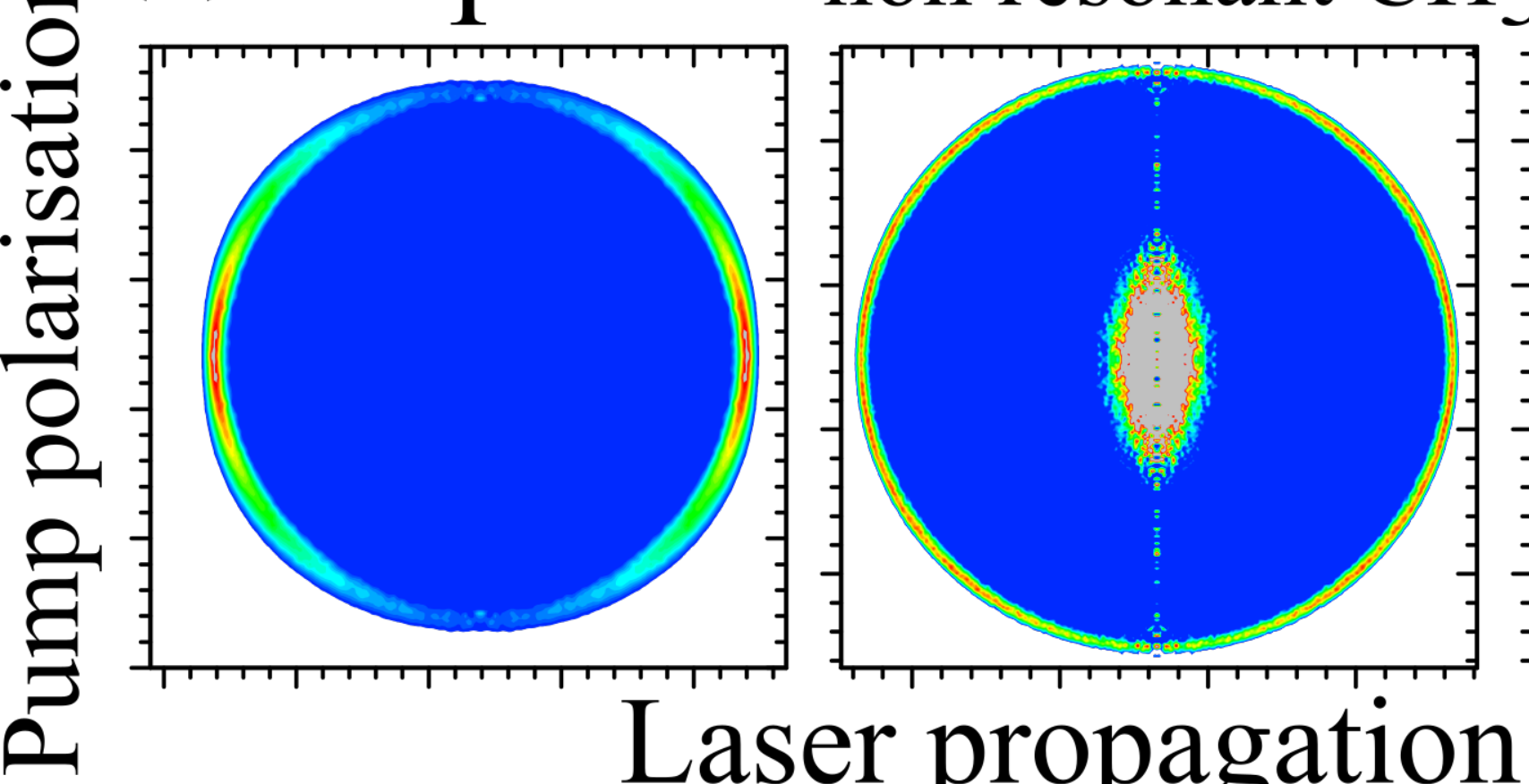

Laser propagation axis

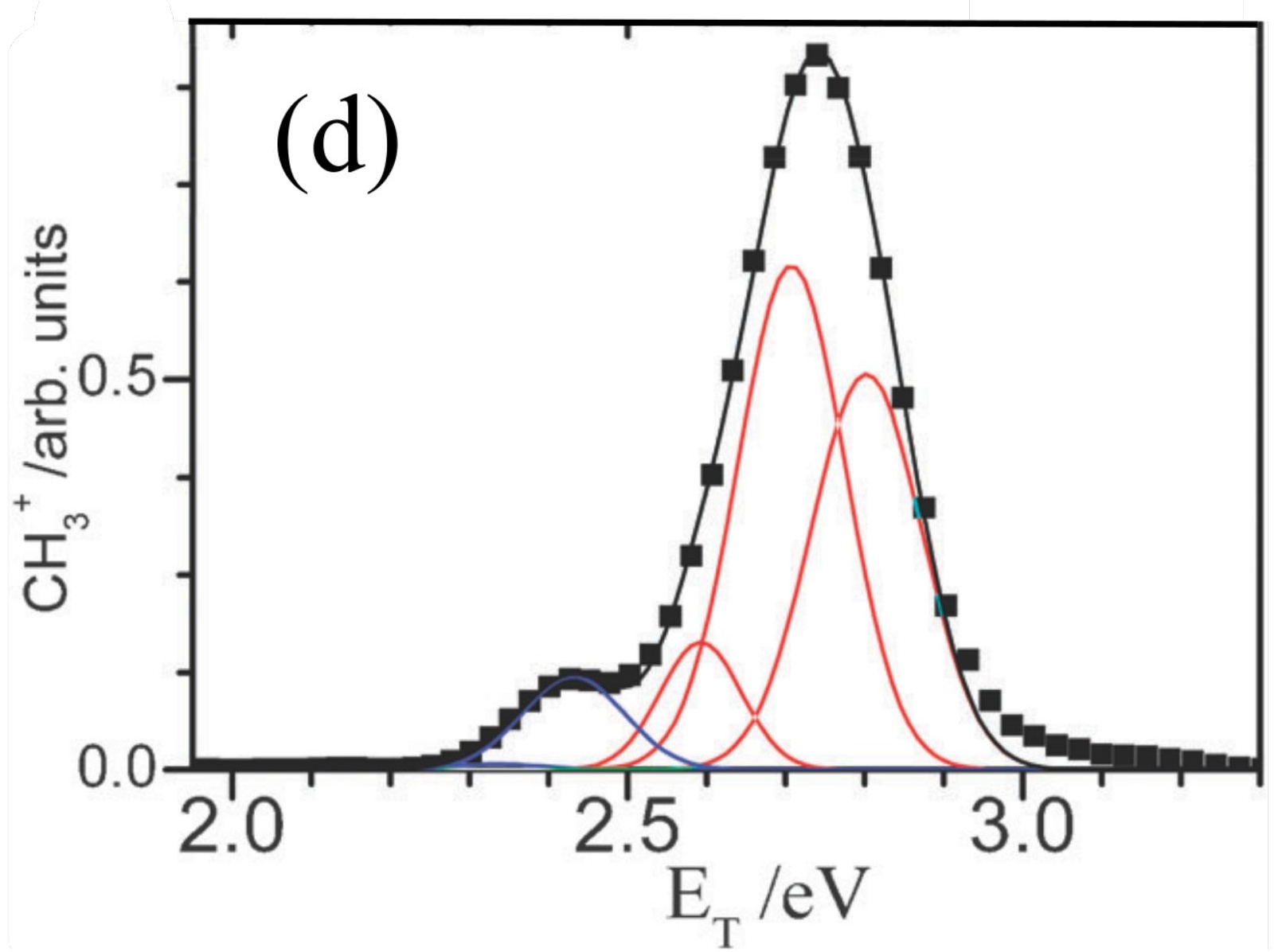

